\title{
Review Article \\ Foundations of the Theory of Semilinear Stochastic Partial Differential Equations
}

\author{
Stefan Tappe \\ Leibniz Universität Hannover, Institut für Mathematische Stochastik, Welfengarten 1, 30167 Hannover, Germany \\ Correspondence should be addressed to Stefan Tappe; tappe@stochastik.uni-hannover.de
}

Received 28 May 2013; Accepted 16 August 2013

Academic Editor: Hong-Kun Xu

Copyright (c) 2013 Stefan Tappe. This is an open access article distributed under the Creative Commons Attribution License, which permits unrestricted use, distribution, and reproduction in any medium, provided the original work is properly cited.

\begin{abstract}
The goal of this review article is to provide a survey about the foundations of semilinear stochastic partial differential equations. In particular, we provide a detailed study of the concepts of strong, weak, and mild solutions, establish their connections, and review a standard existence and uniqueness result. The proof of the existence result is based on a slightly extended version of the Banach fixed point theorem.
\end{abstract}

\section{Introduction}

Semilinear stochastic partial differential equations (SPDEs) have a broad spectrum of applications including natural sciences and economics. The goal of this review article is to provide a survey on the foundations of SPDEs, which have been presented in the monographs [1-3]. It may be beneficial for students who are already aware about stochastic calculus in finite dimensions and who wish to have survey material accompanying the aforementioned references. In particular, we review the relevant results from functional analysis about unbounded operators in Hilbert spaces and strongly continuous semigroups.

A large part of this paper is devoted to a detailed study of the concepts of strong, weak, and mild solutions to SPDEs, to establish their connections and to review and prove a standard existence and uniqueness result. The proof of the existence result is based on a slightly extended version of the Banach fixed point theorem.

In the last part of this paper, we study invariant manifolds for weak solutions to SPDEs. This topic does not belong to the general theory of SPDEs, but it uses and demonstrates many of the results and techniques of the previous sections. It arises from the natural desire to express the solutions of SPDEs, which generally live in an infinite dimensional state space, by means of a finite dimensional state process and thus to ensure larger analytical tractability.
This paper should also serve as an introductory study to the general theory of SPDEs, and it should enable the reader to learn about further topics and generalizations in this field. Possible further directions are the study of martingale solutions (see, e.g., [1, 3]), SPDEs with jumps (see, e.g., [4] for SPDEs driven by the Lévy processes and [5-8] for SPDEs driven by Poisson random measures), and support theorems as well as further invariance results for SPDEs; see, for example, $[9,10]$.

The remainder of this paper is organized as follows: In Sections 2 and 3, we review the required results from functional analysis. In particular, we collect the relevant material about unbounded operators and strongly continuous semigroups. In Section 4 we review stochastic processes in infinite dimension. In particular, we recall the definition of a trace class Wiener process and outline the construction of the Itô integral. In Section 5 we present the solution concepts for SPDEs and study their various connections. In Section 6 we review results about the regularity of stochastic convolution integrals, which is essential for the study of mild solutions to SPDEs. In Section 7 we review a standard existence and uniqueness result. Finally, in Section 8 we deal with invariant manifolds for weak solutions to SPDEs.

\section{Unbounded Operators in the Hilbert Spaces}

In this section, we review the relevant properties about unbounded operators. We will start with operators in Banach 
spaces and focus on operators in Hilbert spaces later on. The reader can find the proofs of the upcoming results in any textbook about functional analysis, such as [11] or [12].

Let $X$ and $Y$ be Banach spaces. For a linear operator $A$ : $X \supset \mathscr{D}(A) \rightarrow Y$, defined on some subspace $\mathscr{D}(A)$ of $X$, we call $\mathscr{D}(A)$ the domain of $A$.

Definition 1. A linear operator $A: X \supset \mathscr{D}(A) \rightarrow Y$ is called closed, if for every sequence $\left(x_{n}\right)_{n \in \mathbb{N}} \subset \mathscr{D}(A)$, such that the limits $x=\lim _{n \rightarrow \infty} x_{n} \in X$ and $y=\lim _{n \rightarrow \infty} A x_{n} \in Y$ exist, one has $x \in \mathscr{D}(A)$ and $A x=y$.

Definition 2. A linear operator $A: X \supset \mathscr{D}(A) \rightarrow Y$ is called densely defined, if its domain $\mathscr{D}(A)$ is dense in $X$; that is, $\overline{\mathscr{D}(A)}=X$.

Definition 3. Let $A: X \supset \mathscr{D}(A) \rightarrow X$ be a linear operator.

(1) The resolvent set of $A$ is defined as

$$
\begin{gathered}
\rho(A):=\{\lambda \in \mathbb{C}: \lambda-A: \mathscr{D}(A) \longrightarrow X \text { is bijective and } \\
\left.(\lambda-A)^{-1} \in L(X)\right\} .
\end{gathered}
$$

(2) The spectrum of $A$ is defined as $\sigma(A):=\mathbb{C} \backslash \rho(A)$.

(3) For $\lambda \in \rho(A)$, one defines the resolvent $R(\lambda, A) \in$ $L(X)$ as

$$
R(\lambda, A):=(\lambda-A)^{-1}
$$

Now, we will introduce the adjoint operator of a densely defined operator in a Hilbert space. Recall that for a bounded linear operator $T \in L\left(H_{1}, H_{2}\right)$, mapping between two Hilbert spaces $H_{1}$ and $H_{2}$, the adjoint operator is the unique bounded linear operator $T^{*} \in L\left(H_{2}, H_{1}\right)$ such that

$$
\langle T x, y\rangle_{H_{2}}=\left\langle x, T^{*} y\right\rangle_{H_{1}} \quad \forall x \in H_{1}, y \in H_{2} .
$$

In order to extend this definition to unbounded operators, one recalls the following extension result for linear operators.

Proposition 4. Let $X$ be a normed space, let $Y$ be a Banach space, let $D \subset X$ be a dense subspace, and let $\Phi: D \rightarrow Y$ be a continuous linear operator. Then there exists a unique continuous extension $\widehat{\Phi}: X \rightarrow Y$, that is, a continuous linear operator with $\left.\widehat{\Phi}\right|_{D}=\Phi$. Moreover, one has $\|\widehat{\Phi}\|=\|\Phi\|$.

Now, let $H$ be a Hilbert space. We recall the representation theorem of Fréchet-Riesz. In the sequel, the space $H^{\prime}$ denotes the dual space of $H$.

Theorem 5. For every $x^{\prime} \in H^{\prime}$, there exists a unique element $x \in H$ with $\left\langle x^{\prime}, \bullet\right\rangle=\langle x, \bullet\rangle$. In addition, one has $\|x\|=\left\|x^{\prime}\right\|$.

Let $A: H \supset \mathscr{D}(A) \rightarrow H$ be a densely defined operator. One defines the subspace

$$
\begin{gathered}
\mathscr{D}\left(A^{*}\right):=\{y \in H: x \longmapsto\langle A x, y\rangle \text { is continuous } \\
\text { on } \mathscr{D}(A)\} .
\end{gathered}
$$

Let $y \in \mathscr{D}\left(A^{*}\right)$ be arbitrary. By virtue of the extension result for linear operators (Proposition 4), the operator

$$
\mathscr{D}(A) \longmapsto \mathbb{R}, \quad x \longmapsto\langle A x, y\rangle,
$$

has a unique extension to a linear functional $z^{\prime} \in H^{\prime}$. By the representation theorem of Fréchet-Riesz (Theorem 5), there exists a unique element $z \in H$ with $\left\langle z^{\prime}, \bullet\right\rangle=\langle z, \bullet\rangle$. This implies that

$$
\langle A x, y\rangle=\langle x, z\rangle \quad \forall x \in \mathscr{D}(A)
$$

Setting $A^{*} y:=z$, this defines a linear operator $A^{*}: H \supset$ $\mathscr{D}\left(A^{*}\right) \rightarrow H$, and one has

$$
\langle A x, y\rangle=\left\langle x, A^{*} y\right\rangle \quad \forall x \in \mathscr{D}(A), y \in \mathscr{D}\left(A^{*}\right) .
$$

Definition 6. The operator $A^{*}: H \supset \mathscr{D}\left(A^{*}\right) \rightarrow H$ is called the adjoint operator of $A$.

Proposition 7. Let $A: H \supset \mathscr{D}(A) \rightarrow H$ be densely defined and closed. Then $A^{*}$ is densely defined and one has $A=A^{* *}$.

Lemma 8. Let $H$ be a separable Hilbert space and let $A$ : $H \supset \mathscr{D}(A) \rightarrow H$ be a closed operator. Then the domain $\left(\mathscr{D}(A),\|\cdot\|_{\mathscr{D}(A)}\right)$ endowed with the graph norm

$$
\|x\|_{\mathscr{D}(A)}=\left(\|x\|^{2}+\|A x\|^{2}\right)^{1 / 2}
$$

is a separable Hilbert space, too.

\section{Strongly Continuous Semigroups}

In this section, we present the required results about strongly continuous semigroups. Concerning the proofs of the upcoming results, the reader is referred to any textbook about functional analysis, such as [11] or [12]. Throughout this section, let $X$ be a Banach space.

Definition 9. Let $\left(S_{t}\right)_{t \geq 0}$ be a family of continuous linear operators $S_{t}: X \rightarrow X, t \geq 0$.

(1) The family $\left(S_{t}\right)_{t \geq 0}$ is a called a strongly continuous semigroup (or $C_{0}$-semigroup), if the following conditions are satisfied:

(i) $S_{0}=I d$,

(ii) $S_{s+t}=S_{s} S_{t}$ for all $s, t \geq 0$,

(iii) $\lim _{t \rightarrow 0} S_{t} x=x$ for all $x \in X$.

(2) The family $\left(S_{t}\right)_{t \geq 0}$ is called a norm continuous semigroup, if the following conditions are satisfied:

(i) $S_{0}=$ Id,

(ii) $S_{s+t}=S_{s} S_{t}$ for all $s, t \geq 0$,

(iii) $\lim _{t \rightarrow 0}\left\|S_{t}-\mathrm{Id}\right\|=0$.

Note that every norm continuous semigroup is also a $C_{0^{-}}$ semigroup. The following growth estimate (9) will often be used when dealing with SPDEs. 
Lemma 10. Let $\left(S_{t}\right)_{t \geq 0}$ be a $C_{0}$-semigroup. Then there are constants $M \geq 1$ and $\omega \in \mathbb{R}$ such that

$$
\left\|S_{t}\right\| \leq M e^{\omega t} \quad \forall t \geq 0 .
$$

Definition 11. Let $\left(S_{t}\right)_{t \geq 0}$ be a $C_{0}$-semigroup.

(1) The semigroup $\left(S_{t}\right)_{t \geq 0}$ is called a semigroup of contractions (or contractive), if

$$
\left\|S_{t}\right\| \leq 1 \quad \forall t \geq 0
$$

that is, the growth estimate (9) is satisfied with $M=1$ and $\omega=0$.

(2) The semigroup $\left(S_{t}\right)_{t \geq 0}$ is called a semigroup of pseudocontractions (or pseudocontractive), if there exists a constant $\omega \in \mathbb{R}$ such that

$$
\left\|S_{t}\right\| \leq e^{\omega t} \quad \forall t \geq 0
$$

that is, the growth estimate (9) is satisfied with $M=1$.

If $\left(S_{t}\right)_{t \geq 0}$ is a semigroup of pseudocontractions with growth estimate (11), then $\left(T_{t}\right)_{t \geq 0}$ given by

$$
T_{t}:=e^{-\omega t} S_{t}, \quad t \geq 0,
$$

is a semigroup of contractions. Hence, every pseudocontractive semigroup can be transformed into a semigroup of contractions, which explains the term pseudocontractive.

Lemma 12. Let $\left(S_{t}\right)_{t \geq 0}$ be a $C_{0}$-semigroup. Then the following statements are true.

(1) The mapping

$$
\mathbb{R}_{+} \times X \longrightarrow X, \quad(t, x) \longmapsto S_{t} x,
$$

is continuous.

(2) For all $x \in X$ and $T \geq 0$, the mapping

$$
[0, T] \longrightarrow X, \quad t \longmapsto S_{t} x,
$$

is uniformly continuous.

Definition 13. Let $\left(S_{t}\right)_{t \geq 0}$ be a $C_{0}$-semigroup. The infinitesimal generator (in short generator) of $\left(S_{t}\right)_{t \geq 0}$ is the linear operator $A: X \supset \mathscr{D}(A) \rightarrow X$, which is defined on the domain

$$
\mathscr{D}(A):=\left\{x \in X: \lim _{t \rightarrow 0} \frac{S_{t} x-x}{t} \text { exists }\right\}
$$

and given by

$$
A x:=\lim _{t \rightarrow 0} \frac{S_{t} x-x}{t} .
$$

Note that the domain $\mathscr{D}(A)$ is indeed a subspace of $X$. The following result gives some properties of the infinitesimal generator of a $C_{0}$-semigroup. Recall that we have provided the required concepts in Definitions 1 and 2.
Proposition 14. The infinitesimal generator $A: X>$ $\mathscr{D}(A) \rightarrow X$ of a $C_{0}$-semigroup $\left(S_{t}\right)_{t \geq 0}$ is densely defined and closed.

We proceed with some examples of $C_{0}$-semigroups and their generators.

Example 15. For every bounded linear operator $A \in L(X)$ the family $\left(e^{t A}\right)_{t \geq 0}$ given by

$$
e^{t A}:=\sum_{n=0}^{\infty} \frac{t^{n} A^{n}}{n !}
$$

is a norm continuous semigroup with generator $A$. In particular, one has $\mathscr{D}(A)=X$.

Example 16. We consider the separable Hilbert space $X=$ $L^{2}(\mathbb{R})$. Let $\left(S_{t}\right)_{t \geq 0}$ be the shift semigroup that is defined as

$$
S_{t} f:=f(t+\bullet), \quad t \geq 0 .
$$

Then $\left(S_{t}\right)_{t \geq 0}$ is a semigroup of contractions with generator $A$ : $L^{2}(\mathbb{R}) \supset \mathscr{D}(A) \rightarrow L^{2}(\mathbb{R})$ given by

$$
\begin{gathered}
\mathscr{D}(A)=\left\{f \in L^{2}(\mathbb{R}): f\right. \text { is absolutely continuous } \\
\text { and } \left.f^{\prime} \in L^{2}(\mathbb{R})\right\}, \\
A f=f^{\prime} .
\end{gathered}
$$

Example 17. On the separable Hilbert space $X=L^{2}\left(\mathbb{R}^{d}\right)$ we define the heat semigroup $\left(S_{t}\right)_{t \geq 0}$ by $S_{0}:=$ Id and

$$
\begin{array}{r}
\left(S_{t} f\right)(x):=\frac{1}{(4 \pi t)^{d / 2}} \int_{\mathbb{R}^{d}} \exp \left(-\frac{|x-y|^{2}}{4 t}\right) f(y) d y \\
t>0 ;
\end{array}
$$

that is, $S_{t} f$ arises as the convolution of $f$ with the density of the normal distribution $N(0,2 t)$. Then $\left(S_{t}\right)_{t \geq 0}$ is a semigroup of contractions with generator $A: L^{2}\left(\mathbb{R}^{d}\right) \supset \mathscr{D}(A) \rightarrow$ $L^{2}\left(\mathbb{R}^{d}\right)$ given by

$$
\mathscr{D}(A)=W^{2}\left(\mathbb{R}^{d}\right), \quad A f=\Delta f .
$$

Here, $W^{2}\left(\mathbb{R}^{d}\right)$ denotes the Sobolev space

$$
\begin{gathered}
W^{2}\left(\mathbb{R}^{d}\right)=\left\{f \in L^{2}\left(\mathbb{R}^{d}\right): D^{(\alpha)} f \in L^{2}\left(\mathbb{R}^{d}\right)\right. \text { exists } \\
\left.\forall \alpha \in \mathbb{N}_{0}^{d} \text { with }|\alpha| \leq 2\right\}
\end{gathered}
$$

and $\Delta$ the Laplace operator

$$
\Delta=\sum_{i=1}^{d} \frac{\partial^{2}}{\partial x_{i}^{2}} .
$$

We proceed with some results regarding calculations with strongly continuous semigroups and their generators. 
Lemma 18. Let $\left(S_{t}\right)_{t \geq 0}$ be a $C_{0}$-semigroup with infinitesimal generator $A$. Then the following statements are true.

(1) For every $x \in \mathscr{D}(A)$, the mapping

$$
\mathbb{R}_{+} \longrightarrow X, \quad t \longmapsto S_{t} x
$$

belongs to class $C^{1}\left(\mathbb{R}_{+} ; X\right)$, and for all $t \geq 0$, one has $S_{t} x \in \mathscr{D}(A)$ and

$$
\frac{d}{d t} S_{t} x=A S_{t} x=S_{t} A x
$$

(2) For all $x \in X$ and $t \geq 0$, one has $\int_{0}^{t} S_{s} x d s \in \mathscr{D}(A)$ and

$$
A\left(\int_{0}^{t} S_{s} x d s\right)=S_{t} x-x
$$

(3) For all $x \in \mathscr{D}(A)$ and $t \geq 0$, one has

$$
\int_{0}^{t} S_{s} A x d s=S_{t} x-x
$$

The following result shows that the strongly continuous semigroup $\left(S_{t}\right)_{t \geq 0}$ associated with generator $A$ is unique. This explains the term generator.

Proposition 19. Two $C_{0}$-semigroups $\left(S_{t}\right)_{t \geq 0}$ and $\left(T_{t}\right)_{t \geq 0}$ with the same infinitesimal generator $A$ coincide; that is, one has $S_{t}=T_{t}$ for all $t \geq 0$.

The next result characterizes all norm continuous semigroups in terms of their generators.

Proposition 20. Let $\left(S_{t}\right)_{t \geq 0}$ be a $C_{0}$-semigroup with infinitesimal generator $A$. Then the following statements are equivalent.

(1) The semigroup $\left(S_{t}\right)_{t \geq 0}$ is norm continuous.

(2) The operator $A$ is continuous.

(3) The domain of $A$ is given by $\mathscr{D}(A)=X$.

If the previous conditions are satisfied, then one has $S_{t}=e^{t A}$ for all $t \geq 0$.

Now, we are interested in characterizing all linear operators $A$ which are the infinitesimal generator of some strongly continuous semigroup $\left(S_{t}\right)_{t \geq 0}$. The following theorem of Hille-Yosida gives a characterization in terms of the resolvent, which we have introduced in Definition 3.

Theorem 21 (Hille-Yosida theorem). Let $A: X \supset \mathscr{D}(A) \rightarrow$ $X$ be a linear operator and let $M \geq 1, \omega \in \mathbb{R}$ be constants. Then the following statements are equivalent.

(1) $A$ is the generator of a $C_{0}$-semigroup $\left(S_{t}\right)_{t \geq 0}$ with growth estimate (9).

(2) $A$ is densely defined and closed and one has $(\omega, \infty) \subset$ $\rho(A)$ and

$$
\left\|R(\lambda, A)^{n}\right\| \leq M(\lambda-\omega)^{-n} \quad \forall \lambda \in(\omega, \infty), n \in \mathbb{N} .
$$

In particular, we obtain the following characterization of the generators of semigroups of contractions.

Corollary 22. For a linear operator $A: X \supset \mathscr{D}(A) \rightarrow X$ the following statements are equivalent.

(1) A is the generator of a semigroup $\left(S_{t}\right)_{t \geq 0}$ of contractions.

(2) $A$ is densely defined and closed, and one has $(0, \infty) \subset$ $\rho(A)$ and

$$
\|R(\lambda, A)\| \leq \frac{1}{\lambda} \quad \forall \lambda \in(0, \infty) .
$$

Proposition 23. Let $\left(S_{t}\right)_{t \geq 0}$ be a $C_{0}$-semigroup on $X$ with generator $A$. Then the family $\left(\left.S_{t}\right|_{\mathscr{D}(A)}\right)_{t \geq 0}$ is a $C_{0}$-semigroup on $\left(\mathscr{D}(A),\|\cdot\|_{\mathscr{D}(A)}\right)$ with generator $A: \mathscr{D}\left(A^{2}\right) \subset \mathscr{D}(A) \rightarrow$ $\mathscr{D}\left(A^{2}\right)$, where the domain is given by

$$
\mathscr{D}\left(A^{2}\right)=\{x \in \mathscr{D}(A): A x \in \mathscr{D}(A)\} .
$$

Recall that we have introduced the adjoint operator for operators in the Hilbert spaces in Definition 6.

Proposition 24. Let $H$ be a Hilbert space and let $\left(S_{t}\right)_{t \geq 0}$ be a $C_{0}$-semigroup on $H$ with generator $A$. Then the family of adjoint operators $\left(S_{t}^{*}\right)_{t \geq 0}$ is a $C_{0}$-semigroup on $H$ with generator $A^{*}$.

\section{Stochastic Processes in Infinite Dimension}

In this section, we recall the required foundations about stochastic processes in infinite dimension. In particular, we recall the definition of a trace class Wiener process and outline the construction of the Itô integral.

In the sequel, $\left(\Omega, \mathscr{F},\left(\mathscr{F}_{t}\right)_{t \geq 0}, \mathbb{P}\right)$ denotes a filtered probability space satisfying the usual conditions. Let $\mathbb{W}$ be a separable Hilbert space and let $Q \in L(\mathbb{H})$ be a nuclear, selfadjoint, positive definite linear operator.

Definition 25. A $\mathbb{H}$-valued, adapted, continuous process $W$ is called a $Q$-Wiener process, if the following conditions are satisfied.

(i) One has $W_{0}=0$.

(ii) The random variable $W_{t}-W_{s}$ and the $\sigma$-algebra $\mathscr{F}_{s}$ are independent for all $0 \leq s \leq t$.

(iii) One has $W_{t}-W_{s} \sim N(0,(t-s) Q)$ for all $0 \leq s \leq t$.

In Definition 25, the distribution $N(0,(t-s) Q)$ is a Gaussian measure with mean 0 and covariance operator $(t-$ $s) Q ;$ see, for example, [1, Section 2.3.2]. The operator $Q$ is also called the covariance operator of the Wiener process $W$. As $Q$ is a trace class operator, we also call $W$ a trace class Wiener process.

Now, let $W$ be a $Q$-Wiener process. Then, there exist an orthonormal basis $\left(e_{j}\right)_{j \in \mathbb{N}}$ of $\mathbb{U}$ and a sequence $\left(\lambda_{j}\right)_{j \in \mathbb{N}} \subset$ $(0, \infty)$ with $\sum_{j \in \mathbb{N}} \lambda_{j}<\infty$ such that

$$
Q u=\sum_{j \in \mathbb{N}} \lambda_{j}\left\langle u, e_{j}\right\rangle_{\mathbb{H}} e_{j}, \quad u \in \mathbb{H} .
$$


Namely, the $\lambda_{j}$ are the eigenvalues of $Q$, and each $e_{j}$ is an eigenvector corresponding to $\lambda_{j}$. The space $\mathbb{H}_{0}:=Q^{1 / 2}(\mathbb{H})$, equipped with the inner product

$$
\langle u, v\rangle_{\mathbb{E}_{0}}:=\left\langle Q^{-1 / 2} u, Q^{-1 / 2} v\right\rangle_{\mathbb{W}},
$$

is another separable Hilbert space, and $\left(\sqrt{\lambda_{j}} e_{j}\right)_{j \in \mathbb{N}}$ is an orthonormal basis. According to [1, Proposition 4.1], the sequence of stochastic processes $\left(\beta^{j}\right)_{j \in \mathbb{N}}$ defined as

$$
\beta^{j}:=\frac{1}{\sqrt{\lambda_{j}}}\left\langle W, e_{j}\right\rangle_{\mathbb{Q}}, \quad j \in \mathbb{N},
$$

is a sequence of real-valued independent standard Wiener processes, and one has the expansion

$$
W=\sum_{j \in \mathbb{N}} \sqrt{\lambda_{j}} \beta^{j} e_{j}
$$

Now, let us briefly sketch the construction of the Itô integral with respect to the Wiener process $W$. Further details can be found in $[1,3]$. We denote by $L_{2}^{0}(H):=L_{2}\left(\mathbb{H}_{0}, H\right)$ the space of Hilbert-Schmidt operators from $\mathbb{M}_{0}$ into $H$, endowed with the Hilbert-Schmidt norm

$$
\|\Phi\|_{L_{2}^{0}(H)}:=\left(\sum_{j \in \mathbb{N}} \lambda_{j}\left\|\Phi e_{j}\right\|^{2}\right)^{1 / 2}, \quad \Phi \in L_{2}^{0}(H),
$$

which itself is a separable Hilbert space. The construction of the Itô integral is divided into three steps as follows.

(1) For every $L(\mathbb{M}, H)$-valued simple process of the form

$$
X=X_{0} \mathbb{1}_{\{0\}}+\sum_{i=1}^{n} X_{i} \mathbb{1}_{\left(t_{i}, t_{i+1}\right]},
$$

with $0=t_{1}<\cdots<t_{n+1}=T$ and $\mathscr{F}_{t_{i}}$-measurable random variables $X_{i}: \Omega \rightarrow L(\mathbb{H}, H)$ for $i=1, \ldots, n$, we set

$$
\int_{0}^{t} X_{s} d W_{s}:=\sum_{i=1}^{n} X_{i}\left(W_{t \wedge t_{i+1}}-W_{t \wedge t_{i}}\right) .
$$

(2) For every predictable $L_{2}^{0}(H)$-valued process $X$ satisfying

$$
\mathbb{E}\left[\int_{0}^{T}\left\|X_{s}\right\|_{L_{2}^{0}(H)}^{2} d s\right]<\infty
$$

we extend the Itô integral $\int_{0}^{t} X_{s} d W_{s}$ by an extension argument for linear operators. In particular, we obtain the Itô isometry as follows:

$$
\mathbb{E}\left[\left\|\int_{0}^{T} X_{s} d W_{s}\right\|^{2}\right]=\mathbb{E}\left[\int_{0}^{T}\left\|X_{s}\right\|_{L_{2}^{0}(H)}^{2} d s\right] .
$$

(3) By localization, we extend the Itô integral $\int_{0}^{t} X_{s} d W_{s}$ for every predictable $L_{2}^{0}(H)$-valued process $X$ satisfying

$$
\mathbb{P}\left(\int_{0}^{t}\left\|\Phi_{s}\right\|_{L_{2}^{0}(H)}^{2} d s<\infty\right)=1 \quad \forall t \geq 0 .
$$

The Itô integral $\left(\int_{0}^{t} X_{s} d W_{s}\right)_{t \geq 0}$ is an $H$-valued, continuous, local martingale, and we have the series expansion

$$
\int_{0}^{t} X_{s} d W_{s}=\sum_{j \in \mathbb{N}} \int_{0}^{t} X_{s}^{j} d \beta_{s}^{j}, \quad t \geq 0,
$$

where $X^{j}:=\sqrt{\lambda_{j}} X e_{j}$ for each $j \in \mathbb{N}$. An indispensable tool for stochastic calculus in infinite dimensions is Itô's formula, which we will recall here.

Theorem 26 (Itô's formula). Let $E$ be another separable Hilbert space, let $f \in C_{b}^{1,2, \text { loc }}\left(\mathbb{R}_{+} \times H ; E\right)$ be a function, and let $X$ be an $H$-valued Itô process of the form

$$
X_{t}=X_{0}+\int_{0}^{t} Y_{s} d s+\int_{0}^{t} Z_{s} d W_{s}, \quad t \geq 0 .
$$

Then $\left(f\left(t, X_{t}\right)\right)_{t \geq 0}$ is an E-valued Itô process, and one has $\mathbb{P}$ almost surely

$$
\begin{aligned}
& f\left(t, X_{t}\right) \\
& =f\left(0, X_{0}\right)+\int_{0}^{t}\left(D_{s} f\left(s, X_{s}\right)+D_{x} f\left(s, X_{s}\right) Y_{s}\right. \\
& \left.+\frac{1}{2} \sum_{j \in \mathbb{N}} D_{x x} f\left(s, X_{s}\right)\left(Z_{s}^{j}, Z_{s}^{j}\right)\right) d s \\
& +\int_{0}^{t} D_{x} f\left(s, X_{s}\right) Z_{s} d W_{s}, \quad t \geq 0,
\end{aligned}
$$

where one uses the notation $Z^{j}:=\sqrt{\lambda_{j}} Z e_{j}$ for each $j \in \mathbb{N}$.

Proof. This result is a consequence of [3, Theorem 2.9].

\section{Solution Concepts for SPDEs}

In this section, we present the concepts of strong, mild, and weak solutions to SPDEs and discuss their relations.

Let $H$ be a separable Hilbert space, and let $\left(S_{t}\right)_{t \geq 0}$ be a $C_{0}$-semigroup on $H$ with infinitesimal generator $A$. Furthermore, let $W$ be a trace class Wiener process on some separable Hilbert space $\mathbb{H}$. We consider the SPDE:

$$
\begin{gathered}
d X_{t}=\left(A X_{t}+\alpha\left(t, X_{t}\right)\right) d t+\sigma\left(t, X_{t}\right) d W_{t} \\
X_{0}=h_{0} .
\end{gathered}
$$

Here $\alpha: \mathbb{R}_{+} \times H \rightarrow H$ and $\sigma: \mathbb{R}_{+} \times H \rightarrow L_{2}^{0}(H)$ are measurable mappings. 
Definition 27. Let $h_{0}: \Omega \rightarrow H$ be a $\mathscr{F}_{0}$-measurable random variable, and let $\tau>0$ be a strictly positive stopping time. Furthermore, let $X=X^{\left(h_{0}\right)}$ be an $H$-valued, continuous, adapted process such that

$$
\begin{aligned}
& \mathbb{P}\left(\int_{0}^{t \wedge \tau}\left(\left\|X_{s}\right\|+\left\|\alpha\left(s, X_{s}\right)\right\|+\left\|\sigma\left(s, X_{s}\right)\right\|_{L_{2}^{0}(H)}^{2}\right) d s<\infty\right) \\
& \quad=1 \quad \forall t \geq 0 .
\end{aligned}
$$

(1) $X$ is called a local strong solution to (44), if

$$
\begin{gathered}
X_{t \wedge \tau} \in \mathscr{D}(A) \quad \forall t \geq 0, \mathbb{P} \text {-almost surely, } \\
\mathbb{P}\left(\int_{0}^{t \wedge \tau}\left\|A X_{s}\right\| d s<\infty\right)=1 \quad \forall t \geq 0,
\end{gathered}
$$

and $\mathbb{P}$-almost surely one has

$$
\begin{aligned}
X_{t \wedge \tau}= & h_{0}+\int_{0}^{t \wedge \tau}\left(A X_{s}+\alpha\left(s, X_{s}\right)\right) d s \\
& +\int_{0}^{t \wedge \tau} \sigma\left(s, X_{s}\right) d W_{s}, \quad t \geq 0 .
\end{aligned}
$$

(2) $X$ is called a local weak solution to (44), if for all $\zeta \epsilon$ $\mathscr{D}\left(A^{*}\right)$ the following equation is fulfilled $\mathbb{P}$-almost surely:

$$
\begin{aligned}
\left\langle\zeta, X_{t \wedge \tau}\right\rangle= & \left\langle\zeta, h_{0}\right\rangle+\int_{0}^{t \wedge \tau}\left(\left\langle A^{*} \zeta, X_{s}\right\rangle+\left\langle\zeta, \alpha\left(s, X_{s}\right)\right\rangle\right) d s \\
& +\int_{0}^{t \wedge \tau}\left\langle\zeta, \sigma\left(s, X_{s}\right)\right\rangle d W_{s}, \quad t \geq 0
\end{aligned}
$$

(3) $X$ is called a local mild solution to (44), if $\mathbb{P}$-almost surely one has

$$
\begin{aligned}
X_{t \wedge \tau}= & S_{t \wedge \tau} h_{0}+\int_{0}^{t \wedge \tau} S_{(t \wedge \tau)-s} \alpha\left(s, X_{s}\right) d s \\
& +\int_{0}^{t \wedge \tau} S_{(t \wedge \tau)-s} \sigma\left(s, X_{s}\right) d W_{s}, \quad t \geq 0 .
\end{aligned}
$$

One calls $\tau$ the lifetime of $X$. If $\tau \equiv \infty$, then one calls $X$ a strong, weak or mild solution to (44), respectively.

Remark 28. Note that the concept of a strong solution is rather restrictive, because condition (46) has to be fulfilled.

For what follows, we fix a $\mathscr{F}_{0}$-measurable random variable $h_{0}: \Omega \rightarrow H$ and a strictly positive stopping time $\tau>0$.

Proposition 29. Every local strong solution $X$ to (44) with lifetime $\tau$ is also a local weak solution to (44) with lifetime $\tau$.
Proof. Let $X$ be a local strong solution to (44) with lifetime $\tau$. Furthermore, let $\zeta \in \mathscr{D}\left(A^{*}\right)$ be arbitrary. Then we have $\mathbb{P}$-almost surely for all $t \geq 0$ the identities

$$
\begin{aligned}
\left\langle\zeta, X_{t \wedge \tau}\right\rangle= & \left\langle\zeta, h_{0}+\int_{0}^{t \wedge \tau}\left(A X_{s}+\alpha\left(s, X_{s}\right)\right) d s\right. \\
& \left.+\int_{0}^{t \wedge \tau} \sigma\left(s, X_{s}\right) d W_{s}\right\rangle \\
= & \left\langle\zeta, h_{0}\right\rangle+\int_{0}^{t \wedge \tau}\left\langle\zeta, A X_{s}+\alpha\left(s, X_{s}\right)\right\rangle d s \\
& +\int_{0}^{t \wedge \tau}\left\langle\zeta, \sigma\left(s, X_{s}\right)\right\rangle d W_{s} \\
= & \left\langle\zeta, h_{0}\right\rangle+\int_{0}^{t \wedge \tau}\left(\left\langle A^{*} \zeta, X_{s}\right\rangle+\left\langle\zeta, \alpha\left(s, X_{s}\right)\right\rangle\right) d s \\
& +\int_{0}^{t \wedge \tau}\left\langle\zeta, \sigma\left(s, X_{s}\right)\right\rangle d W_{s},
\end{aligned}
$$

showing that $X$ is also a local weak solution to (44) with lifetime $\tau$.

Proposition 30. Let $X$ be a stochastic process with $X_{0}=h_{0}$. Then the following statements are equivalent.

(1) The process $X$ is a local strong solution to (44) with lifetime $\tau$.

(2) The process $X$ is a local weak solution to (44) with lifetime $\tau$, and one has (46), (47).

Proof. $(1) \Rightarrow(2)$ : This implication is a direct consequence of Proposition 29.

$(2) \Rightarrow(1)$ : Let $\zeta \in \mathscr{D}\left(A^{*}\right)$ be arbitrary. Then we have $\mathbb{P}$ almost surely for all $t \geq 0$ the identities

$$
\begin{aligned}
\left\langle\zeta, X_{t \wedge \tau}\right\rangle= & \left\langle\zeta, h_{0}\right\rangle+\int_{0}^{t \wedge \tau}\left(\left\langle A^{*} \zeta, X_{s}\right\rangle+\left\langle\zeta, \alpha\left(s, X_{s}\right)\right\rangle\right) d s \\
& +\int_{0}^{t \wedge \tau}\left\langle\zeta, \sigma\left(s, X_{s}\right)\right\rangle d W_{s} \\
= & \left\langle\zeta, h_{0}\right\rangle+\int_{0}^{t \wedge \tau}\left\langle\zeta, A X_{s}+\alpha\left(s, X_{s}\right)\right\rangle d s \\
& +\int_{0}^{t \wedge \tau}\left\langle\zeta, \sigma\left(s, X_{s}\right)\right\rangle d W_{s} \\
= & \left\langle\zeta, h_{0}+\int_{0}^{t \wedge \tau}\left(A X_{s}+\alpha\left(s, X_{s}\right)\right) d s\right. \\
& \left.+\int_{0}^{t \wedge \tau} \sigma\left(s, X_{s}\right) d W_{s}\right\rangle .
\end{aligned}
$$


By Proposition 7, the domain $\mathscr{D}\left(A^{*}\right)$ is dense in $H$, and hence we obtain $\mathbb{P}$-almost surely

$$
\begin{aligned}
X_{t \wedge \tau}= & h_{0}+\int_{0}^{t \wedge \tau}\left(A X_{s}+\alpha\left(s, X_{s}\right)\right) d s \\
& +\int_{0}^{t \wedge \tau} \sigma\left(s, X_{s}\right) d W_{s}, \quad t \geq 0 .
\end{aligned}
$$

Consequently, the process $X$ is also a local strong solution to (44) with lifetime $\tau$.

Corollary 31. Let $\mathscr{M} \subset \mathscr{D}(A)$ be a subset such that $A$ is continuous on $\mathscr{M}$, and let $X$ be a local weak solution to (44) with lifetime $\tau$ such that

$$
X_{t \wedge \tau} \in \mathscr{M} \quad \forall t \geq 0, \mathbb{P} \text {-almost surely. }
$$

Then $X$ is also a local strong solution to (44) with lifetime $\tau$.

Proof. Since $\mathscr{M} \subset \mathscr{D}(A)$, condition (54) implies that (46) is fulfilled. Moreover, by the continuity of $A$ on $\mathscr{M}$, the sample paths of the process $A X$ are $\mathbb{P}$-almost surely continuous; and hence, we obtain (47). Consequently, using Proposition 30, the process $X$ is also a local strong solution to (44) with lifetime $\tau$.

Proposition 32. Every strong solution $X$ to (44) is also a mild solution to (44).

Proof. According to Lemma 8, the domain $\left(\mathscr{D}(A),\|\cdot\|_{\mathscr{D}(A)}\right)$ endowed with the graph norm is a separable Hilbert space, too. Hence, by Lemma 18, for all $t \geq 0$, the function

$$
f:[0, t] \times \mathscr{D}(A) \longrightarrow H, \quad f(s, x):=S_{t-s} x,
$$

belongs to the class $C_{b}^{1,2, \text { loc }}([0, t] \times \mathscr{D}(A) ; H)$ with partial derivatives

$$
\begin{gathered}
D_{t} f(t, x)=-A S_{t-s} x, \\
D_{x} f(t, x)=S_{t-s}, \\
D_{x x} f(t, x)=0 .
\end{gathered}
$$

Hence, by Itô's formula (see Theorem 26) and Lemma 18, we obtain $\mathbb{P}$-almost surely:

$$
\begin{aligned}
X_{t}= & f\left(t, X_{t}\right) \\
= & f\left(0, h_{0}\right)+\int_{0}^{t}\left(D_{s} f\left(s, X_{s}\right)\right. \\
& \left.\quad+D_{x} f\left(s, X_{s}\right)\left(A X_{s}+\alpha\left(s, X_{s}\right)\right)\right) d s \\
& +\int_{0}^{t} D_{x} f\left(s, X_{s}\right) \sigma\left(s, X_{s}\right) d W_{s}
\end{aligned}
$$

$$
\begin{aligned}
= & S_{t} h_{0}+\int_{0}^{t}\left(-A S_{t-s} X_{s}+S_{t-s}\left(A X_{s}+\alpha\left(s, X_{s}\right)\right)\right) d s \\
& +\int_{0}^{t} S_{t-s} \sigma\left(s, X_{s}\right) d W_{s} \\
= & S_{t} h_{0}+\int_{0}^{t} S_{t-s} \alpha\left(s, X_{s}\right) d s \\
& +\int_{0}^{t} S_{t-s} \sigma\left(s, X_{s}\right) d W_{s} .
\end{aligned}
$$

Thus, $X$ is also a mild solution to (44).

We recall the following technical auxiliary result without proof and refer, for example, to [3, Section 3.1].

Lemma 33. Let $T \geq 0$ be arbitrary. Then the linear space

$$
U_{T}:=\operatorname{lin}\left\{g \zeta: g \in C^{1}([0, T] ; \mathbb{R}), \zeta \in \mathscr{D}\left(A^{*}\right)\right\}
$$

is dense in $C^{1}\left([0, T], \mathscr{D}\left(A^{*}\right)\right)$, where $\left(\mathscr{D}\left(A^{*}\right),\|\cdot\|_{\mathscr{D}\left(A^{*}\right)}\right)$ is endowed with the graph norm.

Lemma 34. Let $X$ be a weak solution to (44). Then for all $T \geq$ 0 and all $f \in C^{1}\left([0, T], \mathscr{D}\left(A^{*}\right)\right)$, one has $\mathbb{P}$-almost surely

$$
\begin{aligned}
& \left\langle f(t), X_{t}\right\rangle \\
& =\left\langle f(0), h_{0}\right\rangle \\
& \quad+\int_{0}^{t}\left(\left\langle f^{\prime}(s)+A^{*} f(s), X_{s}\right\rangle+\left\langle f(s), \alpha\left(s, X_{s}\right)\right\rangle\right) d s \\
& \quad+\int_{0}^{t}\left\langle f(s), \sigma\left(s, X_{s}\right)\right\rangle d W_{s}, \quad t \in[0, T] .
\end{aligned}
$$

Proof. By virtue of Lemma 33, it suffices to prove formula (59) for all $f \in U_{T}$. Let $f \in U_{T}$ be arbitrary. Then there are $g_{1}, \ldots, g_{n} \in C^{1}([0, T] ; \mathbb{R})$ and $\zeta_{1}, \ldots, \zeta_{n} \in \mathscr{D}\left(A^{*}\right)$ for some $n \in \mathbb{N}$ such that

$$
f(t)=\sum_{i=1}^{n} g_{i}(t) \zeta_{i}, \quad t \in[0, T]
$$

We define the function

$$
F:[0, T] \times \mathbb{R}^{n} \longrightarrow \mathbb{R}, \quad F(t, x):=\sum_{i=1}^{n} g_{i}(t) x_{i} .
$$

Then, we have $F \in C^{1,2}\left([0, T] \times \mathbb{R}^{n} ; \mathbb{R}\right)$ with partial derivatives

$$
\begin{gathered}
D_{t} F(t, x)=\sum_{i=1}^{n} g_{i}^{\prime}(t) x_{i}, \\
D_{x} F(t, x)=\langle g(t), \bullet\rangle_{\mathbb{R}^{n}}, \\
D_{x x} F(t, x)=0 .
\end{gathered}
$$


8

International Journal of Stochastic Analysis

Since $X$ is a weak solution to (44), the $\mathbb{R}^{n}$-valued process

$$
\langle\zeta, X\rangle:=\left\langle\zeta_{i}, X\right\rangle_{i=1, \ldots, n}
$$

is an Ito process with representation

$$
\begin{aligned}
\left\langle\zeta, X_{t}\right\rangle= & \left\langle\zeta, h_{0}\right\rangle+\int_{0}^{t}\left(\left\langle A^{*} \zeta, X_{s}\right\rangle+\left\langle\zeta, \alpha\left(s, X_{s}\right)\right\rangle\right) d s \\
& +\int_{0}^{t}\left\langle\zeta, \sigma\left(s, X_{s}\right)\right\rangle d W_{s}, \quad t \geq 0 .
\end{aligned}
$$

By Itô's formula (Theorem 26), we obtain $\mathbb{P}$-almost surely

$$
\begin{aligned}
&\left\langle f(t), X_{t}\right\rangle=\left\langle\sum_{i=1}^{n} g_{i}(t) \zeta_{i}, X_{t}\right\rangle=\sum_{i=1}^{n} g_{i}(t)\left\langle\zeta_{i}, X_{t}\right\rangle \\
&= F\left(t,\left\langle\zeta, X_{t}\right\rangle\right)=F\left(0,\left\langle\zeta, h_{0}\right\rangle\right) \\
&+\int_{0}^{t}\left(D_{s} F\left(s,\left\langle\zeta, X_{s}\right\rangle\right)+D_{x} F\left(s,\left\langle\zeta, X_{s}\right\rangle\right)\right. \\
&\left.\times\left(\left\langle A^{*} \zeta, X_{s}\right\rangle+\left\langle\zeta, \alpha\left(s, X_{s}\right)\right\rangle\right)\right) d s \\
&+\int_{0}^{t} D_{x} F\left(s,\left\langle\zeta, X_{s}\right\rangle\right)\left\langle\zeta, \sigma\left(s, X_{s}\right)\right\rangle d W_{s} \\
&= \sum_{i=1}^{n} g_{i}(0)\left\langle\zeta_{i}, h_{0}\right\rangle \\
&+\int_{0}^{t}\left(\sum_{i=1}^{n} g_{i}^{\prime}(t)\left\langle\zeta_{i}, X_{s}\right\rangle\right. \\
&+\int_{0}^{t}\left(\sum_{i=1}^{n} g_{i}(s)\left\langle\zeta_{i}, \sigma\left(s, X_{s}\right)\right\rangle\right) d W_{s}, \\
&+\sum_{i=1}^{n} g_{i}(t)\left(\left\langle A^{*} \zeta_{i}, X_{s}\right\rangle\right. \\
&\left.\left.+\left\langle\zeta_{i}, \alpha\left(s, X_{s}\right)\right\rangle\right)\right) d s
\end{aligned}
$$

and hence

$$
\begin{aligned}
& \left\langle f(t), X_{t}\right\rangle \\
& =\left\langle\sum_{i=1}^{n} g_{i}(0) \zeta_{i}, h_{0}\right\rangle \\
& \quad+\int_{0}^{t}\left(\left\langle\sum_{i=1}^{n} g_{i}^{\prime}(s) \zeta_{i}, X_{s}\right\rangle+\left\langle A^{*}\left(\sum_{i=1}^{n} g_{i}(s) \zeta_{i}\right), X_{s}\right\rangle\right. \\
& \left.\quad+\left\langle\sum_{i=1}^{n} g_{i}(s) \zeta_{i}, \alpha\left(s, X_{s}\right)\right\rangle\right) d s \\
& +\int_{0}^{t}\left\langle\sum_{i=1}^{n} g_{i}(s) \zeta_{i}, \sigma\left(s, X_{s}\right)\right\rangle d W_{s}
\end{aligned}
$$

$$
\begin{array}{r}
=\left\langle f(0), h_{0}\right\rangle+\int_{0}^{t}\left(\left\langle f^{\prime}(s)+A^{*} f(s), X_{s}\right\rangle\right. \\
\left.+\left\langle f(s), \alpha\left(s, X_{s}\right)\right\rangle\right) d s \\
+\int_{0}^{t}\left\langle f(s), \sigma\left(s, X_{s}\right)\right\rangle d W_{s}, \quad t \in[0, T] .
\end{array}
$$

This concludes the proof.

Proposition 35. Every weak solution $X$ to (44) is also a mild solution to (44).

Proof. By Proposition 24, the family $\left(S_{t}^{*}\right)_{t \geq 0}$ is a $C_{0}$-semigroup with generator $A^{*}$. Thus, Proposition 23 yields that the family of restrictions $\left(S_{t}^{*} I_{\mathscr{D}\left(A^{*}\right)}\right)_{t \geq 0}$ is a $C_{0}$-semigroup on $\left(\mathscr{D}\left(A^{*}\right),\|\cdot\|_{\mathscr{D}\left(A^{*}\right)}\right)$ with generator $A^{*}: \mathscr{D}\left(\left(A^{*}\right)^{2}\right) \subset$ $\mathscr{D}\left(A^{*}\right) \rightarrow \mathscr{D}\left(\left(A^{*}\right)^{2}\right)$.

Now, let $t \geq 0$ and $\zeta \in \mathscr{D}\left(\left(A^{*}\right)^{2}\right)$ be arbitrary. We define the function

$$
f:[0, t] \longrightarrow \mathscr{D}\left(A^{*}\right), \quad f(s):=S_{t-s}^{*} \zeta
$$

By Lemma 18 we have $f \in C^{1}\left([0, t] ; \mathscr{D}\left(A^{*}\right)\right)$ with derivative

$$
f^{\prime}(s)=-A^{*} S_{t-s}^{*} \zeta=-A^{*} f(s) .
$$

Using Lemma 34, we obtain $\mathbb{P}$-almost surely

$$
\begin{aligned}
\left\langle\zeta, X_{t}\right\rangle= & \left\langle f(t), X_{t}\right\rangle \\
= & \left\langle f(0), h_{0}\right\rangle+\int_{0}^{t}\left\langle f(s), \alpha\left(s, X_{s}\right)\right\rangle d s \\
& +\int_{0}^{t}\left\langle f(s), \sigma\left(s, X_{s}\right)\right\rangle d W_{s} \\
= & \left\langle S_{t}^{*} \zeta, h_{0}\right\rangle+\int_{0}^{t}\left\langle S_{t-s}^{*} \zeta, \alpha\left(s, X_{s}\right)\right\rangle d s \\
& +\int_{0}^{t}\left\langle S_{t-s}^{*} \zeta, \sigma\left(s, X_{s}\right)\right\rangle d W_{s} \\
= & \left\langle\zeta, S_{t} h_{0}\right\rangle+\int_{0}^{t}\left\langle\zeta, S_{t-s} \alpha\left(s, X_{s}\right)\right\rangle d s \\
& +\int_{0}^{t}\left\langle\zeta, S_{t-s} \sigma\left(s, X_{s}\right)\right\rangle d W_{s} \\
= & \left\langle\zeta, S_{t} h_{0}+\int_{0}^{t} S_{t-s} \alpha\left(s, X_{s}\right) d s\right. \\
& \left.+\int_{0}^{t} S_{t-s} \sigma\left(s, X_{s}\right) d W_{s}\right\rangle .
\end{aligned}
$$


International Journal of Stochastic Analysis

9

Since, by Proposition 14, the domain $\mathscr{D}\left(\left(A^{*}\right)^{2}\right)$ is dense in $\left(\mathscr{D}\left(A^{*}\right),\|\cdot\|_{\mathscr{D}\left(A^{*}\right)}\right)$, we get $\mathbb{P}$-almost surely for all $\zeta \in \mathscr{D}\left(A^{*}\right)$ the identity

$$
\begin{array}{r}
\left\langle\zeta, X_{t}\right\rangle=\left\langle\zeta, S_{t} h_{0}+\int_{0}^{t} S_{t-s} \alpha\left(s, X_{s}\right) d s\right. \\
\left.+\int_{0}^{t} S_{t-s} \sigma\left(s, X_{s}\right) d W_{s}\right\rangle .
\end{array}
$$

Since, by Proposition 14, the domain $\mathscr{D}\left(A^{*}\right)$ is dense in $H$, we obtain $\mathbb{P}$-almost surely

$$
X_{t}=S_{t} h_{0}+\int_{0}^{t} S_{t-s} \alpha\left(s, X_{s}\right) d s+\int_{0}^{t} S_{t-s} \sigma\left(s, X_{s}\right) d W_{s},
$$

proving that $X$ is a mild solution to (44).

Remark 36. Now, the proof of Proposition 32 is an immediate consequence of Propositions 29 and 35.

We have just seen that every weak solution to (44) is also a mild solution. Under the following regularity condition (72), the converse of this statement holds true as well.

Proposition 37. Let $X$ be a mild solution to (44) such that

$$
\mathbb{E}\left[\int_{0}^{T}\left\|\sigma\left(s, X_{s}\right)\right\|_{L_{2}^{0}(H)}^{2} d s\right]<\infty \quad \forall T \geq 0 .
$$

Then $X$ is also a weak solution to (44).

Proof. Let $t \geq 0$ and $\zeta \in \mathscr{D}\left(A^{*}\right)$ be arbitrary. Using Lemma 18, we obtain $\mathbb{P}$-almost surely

$$
\begin{aligned}
\int_{0}^{t} & \left\langle A^{*} \zeta, S_{s} h_{0}\right\rangle d s \\
& =\langle A^{*} \zeta, \underbrace{\int_{0}^{t} S_{s} h_{0} d s}_{\in \mathscr{D}(A)}\rangle=\left\langle\zeta, A\left(\int_{0}^{t} S_{s} h_{0} d s\right)\right\rangle \\
& =\left\langle\zeta, S_{t} h_{0}-h_{0}\right\rangle=\left\langle\zeta, S_{t} h_{0}\right\rangle-\left\langle\zeta, h_{0}\right\rangle .
\end{aligned}
$$

By Fubini's theorem for Bochner integrals (see [3, Section 1.1, page 21]) and Lemma 18. we obtain $\mathbb{P}$-almost surely

$$
\begin{aligned}
\int_{0}^{t} & \left\langle A^{*} \zeta, \int_{0}^{s} S_{s-u} \alpha\left(u, X_{u}\right) d u\right\rangle d s \\
& =\left\langle A^{*} \zeta, \int_{0}^{t}\left(\int_{0}^{s} S_{s-u} \alpha\left(u, X_{u}\right) d u\right) d s\right\rangle \\
& =\left\langle A^{*} \zeta, \int_{0}^{t}\left(\int_{u}^{t} S_{s-u} \alpha\left(u, X_{u}\right) d s\right) d u\right\rangle \\
& =\int_{0}^{t}\left\langle A^{*} \zeta, \int_{u}^{t} S_{s-u} \alpha\left(u, X_{u}\right) d s\right\rangle d u \\
& =\int_{0}^{t}\langle A^{*} \zeta, \underbrace{\int_{0}^{t-s} S_{u} \alpha\left(s, X_{s}\right) d u}_{\in \mathscr{D}(A)}\rangle d s
\end{aligned}
$$

$$
\begin{aligned}
& =\int_{0}^{t}\left\langle\zeta, A\left(\int_{0}^{t-s} S_{u} \alpha\left(s, X_{s}\right) d u\right)\right\rangle d s \\
& =\int_{0}^{t}\left\langle\zeta, S_{t-s} \alpha\left(s, X_{s}\right)-\alpha\left(s, X_{s}\right)\right\rangle d s \\
& =\left\langle\zeta, \int_{0}^{t} S_{t-s} \alpha\left(s, X_{s}\right) d s\right\rangle-\int_{0}^{t}\left\langle\zeta, \alpha\left(s, X_{s}\right)\right\rangle d s .
\end{aligned}
$$

Due to assumption (72), we may use Fubini's theorem for stochastic integrals (see [3, Theorem 2.8]), which, together with Lemma 18 , gives us $\mathbb{P}$-almost surely

$$
\begin{aligned}
\int_{0}^{t} & \left\langle A^{*} \zeta, \int_{0}^{s} S_{s-u} \sigma\left(u, X_{u}\right) d W_{u}\right\rangle d s \\
& =\left\langle A^{*} \zeta, \int_{0}^{t}\left(\int_{0}^{s} S_{s-u} \sigma\left(u, X_{u}\right) d W_{u}\right) d s\right\rangle \\
& =\left\langle A^{*} \zeta, \int_{0}^{t}\left(\int_{u}^{t} S_{s-u} \sigma\left(u, X_{u}\right) d s\right) d W_{u}\right\rangle \\
& =\int_{0}^{t}\left\langle A^{*} \zeta, \int_{u}^{t} S_{s-u} \sigma\left(u, X_{u}\right) d s\right\rangle d W_{u} \\
& =\int_{0}^{t}\langle A^{*} \zeta, \underbrace{\int_{0}^{t-s} S_{u} \sigma\left(s, X_{s}\right) d u}_{0}\rangle d W_{s} \\
& =\int_{0}^{t}\left\langle\zeta, A\left(\int_{0}^{t-s} S_{u} \sigma\left(s, X_{s}\right) d u\right)\right\rangle d W_{s} \\
& =\int_{0}^{t}\left\langle\zeta, S_{t-s} \sigma\left(s, X_{s}\right)-\sigma\left(s, X_{s}\right)\right\rangle d W_{s} \\
& =\left\langle\zeta, \int_{0}^{t} S_{t-s} \sigma\left(s, X_{s}\right) d W_{s}\right\rangle-\int_{0}^{t}\left\langle\zeta, \sigma\left(s, X_{s}\right)\right\rangle d W_{s} .
\end{aligned}
$$

Therefore, and since $X$ is a mild solution to (44), we obtain $\mathbb{P}$-almost surely

$$
\begin{aligned}
& \left\langle\zeta, X_{t}\right\rangle \\
& =\left\langle\zeta, S_{t} h_{0}+\int_{0}^{t} S_{t-s} \alpha\left(s, X_{s}\right) d s+\int_{0}^{t} S_{t-s} \sigma\left(s, X_{s}\right) d W_{s}\right\rangle \\
& =\left\langle\zeta, S_{t} h_{0}\right\rangle+\left\langle\zeta, \int_{0}^{t} S_{t-s} \alpha\left(s, X_{s}\right) d s\right\rangle \\
& \quad+\left\langle\zeta, \int_{0}^{t} S_{t-s} \sigma\left(s, X_{s}\right) d W_{s}\right\rangle
\end{aligned}
$$




$$
\begin{aligned}
= & \left\langle\zeta, h_{0}\right\rangle+\int_{0}^{t}\left\langle A^{*} \zeta, S_{s} h_{0}\right\rangle d s \\
& +\int_{0}^{t}\left\langle A^{*} \zeta, \int_{0}^{s} S_{s-u} \alpha\left(u, X_{u}\right) d u\right\rangle d s \\
& +\int_{0}^{t}\left\langle\zeta, \alpha\left(s, X_{s}\right)\right\rangle d s
\end{aligned}
$$

$$
\begin{aligned}
& +\int_{0}^{t}\left\langle A^{*} \zeta, \int_{0}^{s} S_{s-u} \sigma\left(u, X_{u}\right) d W_{u}\right\rangle d s \\
& +\int_{0}^{t}\left\langle\zeta, \sigma\left(s, X_{s}\right)\right\rangle d W_{s},
\end{aligned}
$$

and hence

$$
\begin{aligned}
\left\langle\zeta, X_{t}\right\rangle= & \left\langle\zeta, h_{0}\right\rangle+\int_{0}^{t}\langle A^{*} \zeta \underbrace{S_{s} h_{0}+\int_{0}^{s} S_{s-u} \alpha\left(u, X_{u}\right) d u+\int_{0}^{s} S_{s-u} \sigma\left(u, X_{u}\right) d W_{u}}_{=X_{s}}\rangle d s \\
& +\int_{0}^{t}\left\langle\zeta, \alpha\left(s, X_{s}\right)\right\rangle d s+\int_{0}^{t}\left\langle\zeta, \sigma\left(s, X_{s}\right)\right\rangle d W_{s} \\
= & \left\langle\zeta, h_{0}\right\rangle+\int_{0}^{t}\left(\left\langle A^{*} \zeta, X_{s}\right\rangle+\left\langle\zeta, \alpha\left(s, X_{s}\right)\right\rangle\right) d s+\int_{0}^{t}\left\langle\zeta, \sigma\left(s, X_{s}\right)\right\rangle d W_{s} .
\end{aligned}
$$

Consequently, the process $X$ is also a weak solution to (44).

Next, we provide conditions which ensure that a mild solution to (44) is also a strong solution.

Proposition 38. Let $X$ be a mild solution to (44) such that $\mathbb{P}$ almost surely one has

$$
X_{s}, \alpha\left(s, X_{s}\right) \in \mathscr{D}(A), \quad \sigma\left(s, X_{s}\right) \in L_{2}^{0}(\mathscr{D}(A)) \quad \forall s \geq 0,
$$

as well as

$$
\begin{gathered}
\mathbb{P}\left(\int_{0}^{t}\left(\left\|X_{s}\right\|_{\mathscr{D}(A)}+\left\|\alpha\left(s, X_{s}\right)\right\|_{\mathscr{D}(A)}\right) d s<\infty\right)=1 \quad \forall t \geq 0 \\
\mathbb{E}\left[\int_{0}^{T}\left\|\sigma\left(s, X_{s}\right)\right\|_{L_{2}^{0}(\mathscr{D}(A))}^{2} d s\right]<\infty \quad \forall T \geq 0
\end{gathered}
$$

Then $X$ is also a strong solution to (44).

Proof. By hypotheses (78) and (79), we have (46) and (47). Let $t \geq 0$ be arbitrary. By Lemma 18, we have

$$
S_{t} h_{0}-h_{0}=\int_{0}^{t} A S_{s} h_{0} d s
$$

Furthermore, by Lemma 18 and Fubini's theorem for Bochner integrals (see [3, Section 1.1, page 21]) we have $\mathbb{P}$-almost surely

$$
\begin{aligned}
& \int_{0}^{t}\left(S_{t-s} \alpha\left(s, X_{s}\right)-\alpha\left(s, X_{s}\right)\right) d s \\
& \quad=\int_{0}^{t}\left(\int_{0}^{t-s} A S_{u} \alpha\left(s, X_{s}\right) d u\right) d s
\end{aligned}
$$

$$
\begin{aligned}
& =\int_{0}^{t}\left(\int_{u}^{t} A S_{s-u} \alpha\left(u, X_{u}\right) d s\right) d u \\
& =\int_{0}^{t}\left(\int_{0}^{s} A S_{s-u} \alpha\left(u, X_{u}\right) d u\right) d s \\
& =\int_{0}^{t} A\left(\int_{0}^{s} S_{s-u} \alpha\left(u, X_{u}\right) d u\right) d s .
\end{aligned}
$$

Due to assumption (80), we may use Fubini's theorem for stochastic integrals (see [3, Theorem 2.8]), which, together with Lemma 18, gives us $\mathbb{P}$-almost surely

$$
\begin{gathered}
\int_{0}^{t}\left(S_{t-s} \sigma\left(s, X_{s}\right)-\sigma\left(s, X_{s}\right)\right) d W_{s} \\
=\int_{0}^{t}\left(\int_{0}^{t-s} A S_{u} \sigma\left(s, X_{s}\right) d u\right) d W_{s} \\
=\int_{0}^{t}\left(\int_{u}^{t} A S_{s-u} \sigma\left(u, X_{u}\right) d s\right) d W_{u} \\
=\int_{0}^{t}\left(\int_{0}^{s} A S_{s-u} \sigma\left(u, X_{u}\right) d W_{u}\right) d s \\
=\int_{0}^{t} A\left(\int_{0}^{s} S_{s-u} \sigma\left(u, X_{u}\right) d W_{u}\right) d s
\end{gathered}
$$


Since $X$ is a mild solution to (44), we have $\mathbb{P}$-almost surely

$$
\begin{aligned}
X_{t}= & S_{t} h_{0}+\int_{0}^{t} S_{t-s} \alpha\left(s, X_{s}\right) d s+\int_{0}^{t} S_{t-s} \sigma\left(s, X_{s}\right) d W_{s} \\
= & h_{0}+\int_{0}^{t} \alpha\left(s, X_{s}\right) d s+\int_{0}^{t} \sigma\left(s, X_{s}\right) d W_{s} \\
& +\left(S_{t} h_{0}-h_{0}\right)+\int_{0}^{t}\left(S_{t-s} \alpha\left(s, X_{s}\right)-\alpha\left(s, X_{s}\right)\right) d s \\
& +\int_{0}^{t}\left(S_{t-s} \sigma\left(s, X_{s}\right)-\sigma\left(s, X_{s}\right)\right) d W_{s},
\end{aligned}
$$

and, hence, combining the latter identities, we obtain $\mathbb{P}$ almost surely

$$
\begin{aligned}
X_{t}= & h_{0}+\int_{0}^{t} \alpha\left(s, X_{s}\right) d s+\int_{0}^{t} \sigma\left(s, X_{s}\right) d W_{s} \\
& +\int_{0}^{t} A S_{s} h_{0} d s+\int_{0}^{t} A\left(\int_{0}^{s} S_{s-u} \alpha\left(u, X_{u}\right) d u\right) d s \\
& +\int_{0}^{t} A\left(\int_{0}^{s} S_{s-u} \sigma\left(u, X_{u}\right) d W_{u}\right) d s,
\end{aligned}
$$

which implies that

$$
\begin{aligned}
X_{t}= & h_{0}+\int_{0}^{t} \alpha\left(s, X_{s}\right) d s+\int_{0}^{t} \sigma\left(s, X_{s}\right) d W_{s} \\
& +\int_{0}^{t} A \underbrace{A\left(S_{s} h_{0}+\int_{0}^{s} S_{s-u} \alpha\left(u, X_{u}\right) d u+\int_{0}^{s} S_{s-u} \sigma\left(u, X_{u}\right) d W_{u}\right)}_{=X_{s}} d s \\
= & h_{0}+\int_{0}^{t}\left(A X_{s}+\alpha\left(s, X_{s}\right)\right) d s+\int_{0}^{t} \sigma\left(s, X_{s}\right) d W_{s} .
\end{aligned}
$$

This proves that $X$ is also a strong solution to (44).

The following result shows that for norm continuous semigroups, the concepts of strong, weak, and mild solutions are equivalent. In particular, this applies for finite dimensional state spaces.

Proposition 39. Suppose that the semigroup $\left(S_{t}\right)_{t \geq 0}$ is norm continuous. Let $X$ be a stochastic process with $X_{0}=h_{0}$. Then the following statements are equivalent.

(1) The process $X$ is a strong solution to (44).

(2) The process $X$ is a weak solution to (44).

(3) The process $X$ is a mild solution to (44).

Proof. $(1) \Rightarrow(2)$ : This implication is a consequence of Proposition 29.

$(2) \Rightarrow(3)$ : This implication is a consequence of Proposition 35.

(3) $\Rightarrow(1)$ : By Proposition 20, we have $A \in L(H)$ and $S_{t}=$ $e^{t A}, t \geq 0$. Furthermore, the family $\left(e^{t A}\right)_{t \in \mathbb{R}}$ is a $C_{0}$-group on $H$. Therefore, and since $X$ is a mild solution to (44), we have $\mathbb{P}$-almost surely

$$
\begin{aligned}
X_{t}= & e^{t A} h_{0}+\int_{0}^{t} e^{(t-s) A} \alpha\left(s, X_{s}\right) d s \\
& +\int_{0}^{t} e^{(t-s) A} \sigma\left(s, X_{s}\right) d W_{s}
\end{aligned}
$$

$$
\begin{aligned}
= & e^{t A} h_{0}+e^{t A} \int_{0}^{t} e^{-s A} \alpha\left(s, X_{s}\right) d s \\
& +e^{t A} \int_{0}^{t} e^{-s A} \sigma\left(s, X_{s}\right) d W_{s}, \quad t \geq 0 .
\end{aligned}
$$

Let $Y$ be the Itô process:

$$
Y_{t}:=\int_{0}^{t} e^{-s A} \alpha\left(s, X_{s}\right) d s+\int_{0}^{t} e^{-s A} \sigma\left(s, X_{s}\right) d W_{s}, \quad t \geq 0 .
$$

Then, we have $\mathbb{P}$-almost surely

$$
X_{t}=e^{t A}\left(h_{0}+Y_{t}\right), \quad t \geq 0,
$$

and, by Lemma 18, we have

$$
e^{t A} h_{0}-h_{0}=\int_{0}^{t} A e^{s A} h_{0} d s .
$$

Defining the function

$$
f: \mathbb{R}_{+} \times H \longrightarrow H, \quad f(s, y):=e^{s A} y,
$$

by Lemma 18 , we have $f \in C_{b}^{1,2, \text { loc }}\left(\mathbb{R}_{+} \times H ; H\right)$ with partial derivatives

$$
\begin{gathered}
D_{s} f(s, y)=A e^{s A} y, \\
D_{y} f(s, y)=e^{s A}, \\
D_{y y} f(s, y)=0 .
\end{gathered}
$$


By Itô's formula (Theorem 26), we get $\mathbb{P}$-almost surely

$$
\begin{aligned}
e^{t A} Y_{t}= & f\left(t, Y_{t}\right) \\
= & f(0,0) \\
& +\int_{0}^{t}\left(D_{s} f\left(s, Y_{s}\right)+D_{y} f\left(s, Y_{s}\right) e^{-s A} \alpha\left(s, X_{s}\right)\right) d s \\
& +\int_{0}^{t} D_{y} f\left(s, Y_{s}\right) e^{-s A} \sigma\left(s, X_{s}\right) d W_{s} \\
= & \int_{0}^{t}\left(A e^{s A} Y_{s}+\alpha\left(s, X_{s}\right)\right) d s+\int_{0}^{t} \sigma\left(s, X_{s}\right) d W_{s} .
\end{aligned}
$$

Combining the previous identities, we obtain $\mathbb{P}$-almost surely

$$
\begin{aligned}
& X_{t}= e^{t A}\left(h_{0}+Y_{t}\right) \\
&= h_{0}+\left(e^{t A} h_{0}-h_{0}\right)+e^{t A} Y_{t} \\
&= h_{0}+\int_{0}^{t} A e^{s A} h_{0} d s+\int_{0}^{t}\left(A e^{s A} Y_{s}+\alpha\left(s, X_{s}\right)\right) d s \\
&+\int_{0}^{t} \sigma\left(s, X_{s}\right) d W_{s} \\
&= h_{0}+\int_{0}^{t}(A \underbrace{e^{s A}\left(h_{0}+Y_{s}\right)}_{=X_{s}}+\alpha\left(s, X_{s}\right)) d s \\
&+\int_{0}^{t} \sigma\left(s, X_{s}\right) d W_{s} \\
&= h_{0}+\int_{0}^{t}\left(A X_{s}+\alpha\left(s, X_{s}\right)\right) d s+\int_{0}^{t} \sigma\left(s, X_{s}\right) d W_{s}, \\
& t \geq 0,
\end{aligned}
$$

proving that $X$ is a strong solution to (44).

\section{Stochastic Convolution Integrals}

In this section, we deal with the regularity of stochastic convolution integrals, which occur when dealing with mild solutions to SPDEs of the type (44).

Let $E$ be a separable Banach space, and let $\left(S_{t}\right)_{t \geq 0}$ be a $C_{0}$ semigroup on $E$. We start with the drift term.

Lemma 40. Let $f: \mathbb{R}_{+} \rightarrow$ E be a measurable mapping such that

$$
\int_{0}^{t}\|f(s)\| d s<\infty \quad \forall t \geq 0
$$

Then the mapping

$$
F: \mathbb{R}_{+} \longrightarrow E, \quad F(t):=\int_{0}^{t} S_{t-s} f(s) d s
$$

is continuous.
Proof. Let $t \in \mathbb{R}_{+}$be arbitrary. It suffices to prove that $F$ is right-continuous and left-continuous in $t$.

(1) Let $\left(t_{n}\right)_{n \in \mathbb{N}} \subset \mathbb{R}_{+}$be a sequence such that $t_{n} \downarrow t$. Then for every $n \in \mathbb{N}$ we have

$$
\begin{aligned}
& \left\|F(t)-F\left(t_{n}\right)\right\| \\
& =\left\|\int_{0}^{t} S_{t-s} f(s) d s-\int_{0}^{t_{n}} S_{t_{n}-s} f(s) d s\right\| \\
& =\left\|\int_{0}^{t} S_{t-s} f(s) d s-\int_{0}^{t} S_{t_{n}-s} f(s) d s-\int_{t}^{t_{n}} S_{t_{n}-s} f(s) d s\right\| \\
& \leq \int_{0}^{t}\left\|S_{t-s} f(s)-S_{t_{n}-s} f(s)\right\| d s \\
& \quad+\int_{t}^{t_{n}}\left\|S_{t_{n}-s} f(s)\right\| d s .
\end{aligned}
$$

By Lemma 12, the mapping

$$
\mathbb{R}_{+} \times E \longrightarrow E, \quad(u, x) \longmapsto S_{u} x,
$$

is continuous. Thus, taking into account estimate (9) from Lemma 10, by Lebesgue's dominated convergence theorem we obtain

$$
\left\|F(t)-F\left(t_{n}\right)\right\| \longrightarrow 0 \text { for } n \longrightarrow \infty .
$$

(2) Let $\left(t_{n}\right)_{n \in \mathbb{N}} \subset \mathbb{R}_{+}$be a sequence such that $t_{n} \uparrow t$. Then for every $n \in \mathbb{N}$ we have

$$
\begin{aligned}
\left\|F(t)-F\left(t_{n}\right)\right\| \\
=\left\|\int_{0}^{t} S_{t-s} f(s) d s-\int_{0}^{t_{n}} S_{t_{n}-s} f(s) d s\right\| \\
=\left\|\int_{0}^{t_{n}} S_{t-s} f(s) d s-\int_{t_{n}}^{t} S_{t-s} f(s) d s-\int_{0}^{t_{n}} S_{t_{n}-s} f(s) d s\right\| \\
\leq \int_{0}^{t_{n}}\left\|S_{t-s} f(s)-S_{t_{n}-s} f(s)\right\| d s+\int_{t_{n}}^{t}\left\|S_{t-s} f(s)\right\| d s .
\end{aligned}
$$

Proceeding as in the previous situation, by Lebesgue's dominated convergence theorem we obtain

$$
\left\|F(t)-F\left(t_{n}\right)\right\| \longrightarrow 0 \text { for } n \longrightarrow \infty \text {. }
$$

This completes the proof.

Proposition 41. Let $X$ be a progressively measurable process satisfying

$$
\mathbb{P}\left(\int_{0}^{t}\left\|X_{s}\right\| d s<\infty\right)=1 \quad \forall t \geq 0 .
$$

Then the process $Y$ defined as

$$
Y_{t}:=\int_{0}^{t} S_{t-s} X_{s} d s, \quad t \geq 0,
$$

is continuous and adapted. 
Proof. The continuity of $Y$ is a consequence of Lemma 40. Moreover, $Y$ is adapted, because $X$ is progressively measurable.

Now, we will deal with stochastic convolution integrals driven by the Wiener processes. Let $H$ be a separable Hilbert space, and let $\left(S_{t}\right)_{t \geq 0}$ be a $C_{0}$-semigroup on $H$. Moreover, let $W$ be a trace class Wiener process on some separable Hilbert space $\mathbb{H}$.

Definition 42. Let $X$ be a $L_{2}^{0}(H)$-valued predictable process such that

$$
\mathbb{P}\left(\int_{0}^{t}\left\|X_{s}\right\|_{L_{2}^{0}(H)}^{2} d s<\infty\right)=1 \quad \forall t \geq 0 .
$$

One defines the stochastic convolution $X \star W$ as

$$
(X \star W)_{t}:=\int_{0}^{t} S_{t-s} X_{s} d W_{s}, \quad t \geq 0 .
$$

One recalls the following result concerning the regularity of stochastic convolutions.

Proposition 43. Let $X$ be a $L_{2}^{0}(H)$-valued predictable process such that one of the following two conditions is satisfied.

(1) There exists a constant $p>1$ such that

$$
\mathbb{E}\left[\int_{0}^{t}\left\|X_{s}\right\|_{L_{2}^{0}(H)}^{2 p} d s\right]<\infty \quad \forall t \geq 0 .
$$

(2) The semigroup $\left(S_{t}\right)_{t \geq 0}$ is a semigroup of pseudocontractions, and one has

$$
\mathbb{E}\left[\int_{0}^{t}\left\|X_{s}\right\|_{L_{2}^{0}(H)}^{2} d s\right]<\infty \quad \forall t \geq 0 .
$$

Then the stochastic convolution $X \star W$ has a continuous version.

Proof. See [3, Lemma 3.3].

\section{Existence and Uniqueness Results for SPDEs}

In this section, we will present results concerning existence and uniqueness of solutions to the SPDE (44).

First, we recall the Banach fixed point theorem, which will be a basic result for proving the existence of mild solutions to (44).

Definition 44. Let $(E, d)$ be a metric space, and let $\Phi: E \rightarrow E$ be a mapping.

(1) The mapping $\Phi$ is called a contraction, if for some constant $0 \leq L<1$ one has

$$
d(\Phi(x), \Phi(y)) \leq L \cdot d(x, y) \quad \forall x, y \in E .
$$

(2) An element $x \in E$ is called a fixed point of $\Phi$, if one has

$$
\Phi(x)=x
$$

The following result is the well-known Banach fixed point theorem. Its proof can be found, for example, in [13, Theorem 3.48].

Theorem 45 (The Banach fixed point theorem). Let $E$ be $a$ complete metric space, and let $\Phi: E \rightarrow E$ be a contraction. Then the mapping $\Phi$ has a unique fixed point.

In this text, we will use the following slight extension of the Banach fixed point theorem.

Corollary 46. Let $E$ be a complete metric space, and let $\Phi$ : $E \rightarrow E$ be a mapping such that for some $n \in \mathbb{N}$ the mapping $\Phi^{n}$ is a contraction. Then the mapping $\Phi$ has a unique fixed point.

Proof. According to the Banach fixed point theorem (Theorem 45) the mapping $\Phi^{n}$ has a unique fixed point; that is, there exists a unique element $x \in E$ such that $\Phi^{n}(x)=x$. Therefore, we have

$$
\Phi(x)=\Phi\left(\Phi^{n}(x)\right)=\Phi^{n}(\Phi(x)),
$$

showing that $\Phi(x)$ is a fixed point of $\Phi^{n}$. Since $\Phi^{n}$ has a unique fixed point, we deduce that $\Phi(x)=x$, showing that $x$ is a fixed point of $\Phi$.

In order to prove uniqueness, let $y \in E$ be another fixed point of $\Phi$; that is, we have $\Phi(y)=y$. By induction, we obtain

$$
\Phi^{n}(y)=\Phi^{n-1}(\Phi(y))=\Phi^{n-1}(y)=\cdots=\Phi(y)=y
$$

showing that $y$ is a fixed point of $\Phi^{n}$. Since the mapping $\Phi^{n}$ has exactly one fixed point, we obtain $x=y$.

An indispensable tool for proving uniqueness of mild solutions to (44) will be the following version of Gronwall's inequality; see, for example, [14, Theorem 5.1].

Lemma 47 (Gronwall's inequality). Let $T \geq 0$ be fixed, let $f:[0, T] \rightarrow \mathbb{R}_{+}$be a nonnegative continuous mapping, and let $\beta \geq 0$ be a constant such that

$$
f(t) \leq \beta \int_{0}^{t} f(s) d s \quad \forall t \in[0, T] .
$$

Then one has $f \equiv 0$.

The following result shows that local Lipschitz continuity of $\alpha$ and $\sigma$ ensures the uniqueness of mild solutions to the SPDE (44).

Theorem 48. One supposes that for every $n \in \mathbb{N}$ there exists a constant $L_{n} \geq 0$ such that

$$
\begin{gathered}
\left\|\alpha\left(t, h_{1}\right)-\alpha\left(t, h_{2}\right)\right\| \leq L_{n}\left\|h_{1}-h_{2}\right\|, \\
\left\|\sigma\left(t, h_{1}\right)-\sigma\left(t, h_{2}\right)\right\|_{L_{2}^{0}(H)} \leq L_{n}\left\|h_{1}-h_{2}\right\|,
\end{gathered}
$$

for all $t \geq 0$ and all $h_{1}, h_{2} \in H$ with $\left\|h_{1}\right\|,\left\|h_{2}\right\| \leq n$. Let $h_{0}, g_{0}$ : $\Omega \rightarrow H$ be two $\mathscr{F}_{0}$-measurable random variables, let $\tau>0$ be a strictly positive stopping time, and let $X, Y$ be two local mild 
solutions to (44) with initial conditions $h_{0}, g_{0}$ and lifetime $\tau$. Then one has up to indistinguishability

$$
X^{\tau} \mathbb{1}_{\left\{h_{0}=g_{0}\right\}}=Y^{\tau} \mathbb{1}_{\left\{h_{0}=g_{0}\right\}}
$$

(Two processes $X$ and $Y$ are called indistinguishable if the set $\left\{\omega \in \Omega: X_{t}(\omega) \neq Y_{t}(\omega)\right.$ for some $\left.t \in \mathbb{R}_{+}\right\}$is a $\mathbb{P}$-nullset. $)$

Proof. Defining the stopping times $\left(\tau_{n}\right)_{n \in \mathbb{N}}$ as

$$
\tau_{n}:=\tau \wedge \inf \left\{t \geq 0:\left\|X_{t}\right\| \geq n\right\} \wedge \inf \left\{t \geq 0:\left\|Y_{t}\right\| \geq n\right\}
$$

we have $\mathbb{P}\left(\tau_{n} \rightarrow \tau\right)=1$. Let $n \in \mathbb{N}$ and $T \geq 0$ be arbitrary, and set

$$
\Gamma:=\left\{h_{0}=g_{0}\right\} \in \mathscr{F}_{0} .
$$

The mapping

$$
f:[0, T] \longrightarrow \mathbb{R}, \quad f(t):=\mathbb{E}\left[\mathbb{1}_{\Gamma}\left\|X_{t \wedge \tau_{n}}-Y_{t \wedge \tau_{n}}\right\|^{2}\right],
$$

is nonnegative, and it is continuous by Lebesgue's dominated convergence theorem. For all $t \in[0, T]$ we have

$$
\begin{aligned}
f(t)= & \mathbb{E}\left[\mathbb{1}_{\Gamma}\left\|X_{t \wedge \tau_{n}}-Y_{t \wedge \tau_{n}}\right\|^{2}\right] \\
\leq & \underbrace{3 \mathbb{E}\left[\mathbb{1}_{\Gamma}\left\|S_{t \wedge \tau_{n}}\left(h_{0}-g_{0}\right)\right\|^{2}\right]}_{=0} \\
& +3 \mathbb{E}\left[\mathbb{1}_{\Gamma}\left\|\int_{0}^{t \wedge \tau_{n}} S_{\left(t \wedge \tau_{n}\right)-s}\left(\alpha\left(s, X_{s}\right)-\alpha\left(s, Y_{s}\right)\right) d s\right\|^{2}\right] \\
& +3 \mathbb{E}\left[\mathbb{1}_{\Gamma}\left\|\int_{0}^{t \wedge \tau_{n}} S_{\left(t \wedge \tau_{n}\right)-s}\left(\sigma\left(s, X_{s}\right)-\sigma\left(s, Y_{s}\right)\right) d W_{s}\right\|^{2}\right] \\
= & 3 \mathbb{E}\left[\left\|\int_{0}^{t \wedge \tau_{n}} \mathbb{1}_{\Gamma} S_{\left(t \wedge \tau_{n}\right)-s}\left(\alpha\left(s, X_{s}\right)-\alpha\left(s, Y_{s}\right)\right) d s\right\|^{2}\right] \\
& +3 \mathbb{E}\left[\left\|\int_{0}^{t \wedge \tau_{n}} \mathbb{1}_{\Gamma} S_{\left(t \wedge \tau_{n}\right)-s}\left(\sigma\left(s, X_{s}\right)-\sigma\left(s, Y_{s}\right)\right) d W_{s}\right\|^{2}\right],
\end{aligned}
$$

and hence, by the Cauchy-Schwarz inequality, the Itô isometry (39), the growth estimate (9) from Lemma 10, and the local Lipschitz conditions (113) we obtain

$$
\begin{aligned}
& f(t) \\
& \leq 3 T \mathbb{E}\left[\int_{0}^{t \wedge \tau_{n}}\left\|\mathbb{1}_{\Gamma} S_{\left(t \wedge \tau_{n}\right)-s}\left(\alpha\left(s, X_{s}\right)-\alpha\left(s, Y_{s}\right)\right)\right\|^{2} d s\right] \\
& +3 \mathbb{E}\left[\int_{0}^{t \wedge \tau_{n}}\left\|\mathbb{1}_{\Gamma} S_{\left(t \wedge \tau_{n}\right)-s}\left(\sigma\left(s, X_{s}\right)-\sigma\left(s, Y_{s}\right)\right)\right\|_{L_{2}^{0}(H)}^{2} d s\right] \\
& \leq 3 T\left(M e^{\omega T}\right)^{2} \mathbb{E}\left[\int_{0}^{t \wedge \tau_{n}} \mathbb{1}_{\Gamma}\left\|\alpha\left(s, X_{s}\right)-\alpha\left(s, Y_{s}\right)\right\|^{2} d s\right] \\
& +3\left(M e^{\omega T}\right)^{2} \mathbb{E}\left[\int_{0}^{t \wedge \tau_{n}} \mathbb{1}_{\Gamma}\left\|\sigma\left(s, X_{s}\right)-\sigma\left(s, Y_{s}\right)\right\|_{L_{2}^{0}(H)}^{2} d s\right] \\
& \leq 3(T+1)\left(M e^{\omega T}\right)^{2} L_{n}^{2} \int_{0}^{t} \mathbb{E}\left[\mathbb{1}_{\Gamma}\left\|X_{s \wedge \tau_{n}}-Y_{s \wedge \tau_{n}}\right\|^{2}\right] d s \\
& =3(T+1)\left(M e^{\omega T}\right)^{2} L_{n}^{2} \int_{0}^{t} f(s) d s .
\end{aligned}
$$

Using Gronwall's inequality (see Lemma 47) we deduce that $f \equiv 0$. Thus, by the continuity of the sample paths of $X$ and $Y$, we obtain

$$
\mathbb{P}\left(\bigcap_{t \geq 0}\left\{X_{t \wedge \tau_{n}} \mathbb{1}_{\Gamma}=Y_{t \wedge \tau_{n}} \mathbb{1}_{\Gamma}\right\}\right)=1 \quad \forall n \in \mathbb{N}
$$

and hence, by the continuity of the probability measure $\mathbb{P}$, we conclude that

$$
\begin{aligned}
\mathbb{P}\left(\bigcap_{t \geq 0}\left\{X_{t \wedge \tau} \mathbb{1}_{\Gamma}=Y_{t \wedge \tau} \mathbb{1}_{\Gamma}\right\}\right) \\
\quad=\mathbb{P}\left(\bigcap_{n \in \mathbb{N}} \bigcap_{t \geq 0}\left\{X_{t \wedge \tau_{n}} \mathbb{1}_{\Gamma}=Y_{t \wedge \tau_{n}} \mathbb{1}_{\Gamma}\right\}\right) \\
\quad=\lim _{n \rightarrow \infty} \mathbb{P}\left(\bigcap_{t \geq 0}\left\{X_{t \wedge \tau_{n}} \mathbb{1}_{\Gamma}=Y_{t \wedge \tau_{n}} \mathbb{1}_{\Gamma}\right\}\right)=1,
\end{aligned}
$$

which completes the proof.

The local Lipschitz conditions (113) are, in general, not sufficient in order to ensure the existence of mild solutions to the SPDE (44). Now, we will prove that the existence of mild solutions follows from global Lipschitz and linear growth conditions on $\alpha$ and $\sigma$. For this, we recall an auxiliary result which extends the Itô isometry (39).

Lemma 49. Let $T \geq 0$ be arbitrary, and let $X=\left(X_{t}\right)_{t \in[0, T]}$ be a $L_{2}^{0}(H)$-valued, predictable process such that

$$
\mathbb{E}\left[\int_{0}^{T}\left\|X_{s}\right\|_{L_{2}^{0}(H)}^{2} d s\right]<\infty .
$$


Then, for every $p \geq 1$ one has

$$
\mathbb{E}\left[\left\|\int_{0}^{T} X_{s} d W_{s}\right\|^{2 p}\right] \leq C_{p} \mathbb{E}\left[\int_{0}^{T}\left\|X_{s}\right\|_{L_{2}^{0}(H)}^{2} d s\right]^{p},
$$

where the constant $C_{p}>0$ is given by

$$
C_{p}=(p(2 p-1))^{p}\left(\frac{2 p}{2 p-1}\right)^{2 p^{2}} .
$$

Proof. See [3, Lemma 3.1].

Theorem 50. Suppose that there exists a constant $L \geq 0$ such that

$$
\begin{gathered}
\left\|\alpha\left(t, h_{1}\right)-\alpha\left(t, h_{2}\right)\right\| \leq L\left\|h_{1}-h_{2}\right\|, \\
\left\|\sigma\left(t, h_{1}\right)-\sigma\left(t, h_{2}\right)\right\|_{L_{2}^{0}(H)} \leq L\left\|h_{1}-h_{2}\right\|,
\end{gathered}
$$

for all $t \geq 0$ and all $h_{1}, h_{2} \in H$, and suppose that there exists a constant $K \geq 0$ such that

$$
\begin{gathered}
\|\alpha(t, h)\| \leq K(1+\|h\|), \\
\|\sigma(t, h)\|_{L_{2}^{0}(H)} \leq K(1+\|h\|),
\end{gathered}
$$

for all $t \geq 0$ and all $h \in H$. Then, for every $\mathscr{F}_{0}$-measurable random variable $h_{0}: \Omega \rightarrow H$, there exists a (up to indistinguishability) unique mild solution $X$ to (44).

Proof. The uniqueness of mild solutions to (44) is a direct consequence of Theorem 48, and hence, we may concentrate on the existence proof, which we divide into the following several steps.

Step 1. First, we suppose that the initial condition $h_{0}$ satisfies $\mathbb{E}\left[\left\|h_{0}\right\|^{2 p}\right]<\infty$ for some $p>1$. Let $T \geq 0$ be arbitrary. We define the Banach space

$$
L_{T}^{2 p}(H):=L^{2 p}\left(\Omega \times[0, T], \mathscr{P}_{T}, \mathbb{P} \otimes d t ; H\right),
$$

and prove that the variation of constants equation

$$
\begin{aligned}
X_{t}= & S_{t} h_{0}+\int_{0}^{t} S_{t-s} \alpha\left(s, X_{s}\right) d s \\
& +\int_{0}^{t} S_{t-s} \sigma\left(s, X_{s}\right) d W_{s}, \quad t \in[0, T],
\end{aligned}
$$

has a unique solution in the space $L_{T}^{2 p}(H)$. This is done in the following three steps.

Step 1.1. For $X \in L_{T}^{2 p}(H)$ we define the process $\Phi X$ by

$$
\begin{aligned}
(\Phi X)_{t}= & S_{t} h_{0}+\int_{0}^{t} S_{t-s} \alpha\left(s, X_{s}\right) d s \\
& +\int_{0}^{t} S_{t-s} \sigma\left(s, X_{s}\right) d W_{s}, \quad t \in[0, T] .
\end{aligned}
$$

Then the process $\Phi X$ is well defined. Indeed, by the growth estimate (9), the linear growth condition (127), and Hölder's inequality we have

$$
\begin{aligned}
& \mathbb{E}\left[\int_{0}^{T}\left\|S_{t-s} \alpha\left(s, X_{s}\right)\right\| d s\right] \\
& \leq M e^{\omega T} \mathbb{E}\left[\int_{0}^{T}\left\|\alpha\left(s, X_{s}\right)\right\| d s\right] \\
& \leq M e^{\omega T} K \mathbb{E}\left[\int_{0}^{T}\left(1+\left\|X_{s}\right\|\right) d s\right] \\
& =M e^{\omega T} K\left(T+\mathbb{E}\left[\int_{0}^{T}\left\|X_{s}\right\| d s\right]\right) \\
& \leq M e^{\omega T} K\left(T+T^{1-1 / 2 p}\right. \\
& \left.\times \mathbb{E}\left[\int_{0}^{T}\left\|X_{s}\right\|^{2 p} d s\right]^{1 / 2 p}\right)<\infty .
\end{aligned}
$$

Furthermore, by the growth estimate (9), the linear growth condition (128), and Hölder's inequality we have

$$
\begin{aligned}
& \mathbb{E}\left[\int_{0}^{T}\left\|S_{t-s} \sigma\left(s, X_{s}\right)\right\|_{L_{2}^{0}(H)}^{2} d s\right] \\
& \quad \leq\left(M e^{\omega T}\right)^{2} \mathbb{E}\left[\int_{0}^{T}\left\|\sigma\left(s, X_{s}\right)\right\|_{L_{2}^{0}(H)}^{2} d s\right] \\
& \quad \leq\left(M e^{\omega T} K\right)^{2} \mathbb{E}\left[\int_{0}^{T}\left(1+\left\|X_{s}\right\|\right)^{2} d s\right] \\
& \quad \leq 2\left(M e^{\omega T} K\right)^{2} \mathbb{E}\left[\int_{0}^{T}\left(1+\left\|X_{s}\right\|^{2}\right) d s\right] \\
& \quad=2\left(M e^{\omega T} K\right)^{2}\left(T+\mathbb{E}\left[\int_{0}^{T}\left\|X_{s}\right\|^{2} d s\right]\right) \\
& \quad \leq 2\left(M e^{\omega T} K\right)^{2}\left(T+T^{1-1 / p} \mathbb{E}\left[\int_{0}^{T}\left\|X_{s}\right\|^{2 p}\right]^{1 / p}\right)<\infty .
\end{aligned}
$$

The previous two estimates show that $\Phi$ is a well-defined mapping on $L_{T}^{2 p}(H)$.

Step 1.2. Next, we show that the mapping $\Phi$ maps $L_{T}^{2 p}(H)$ into itself; that is, we have $\Phi: L_{T}^{2 p}(H) \rightarrow L_{T}^{2 p}(H)$. Indeed, let $X \in$ $L_{T}^{2 p}(H)$ be arbitrary. Defining the processes $\Phi_{\alpha} X$ and $\Phi_{\sigma} X$ as

$$
\begin{gathered}
\left(\Phi_{\alpha} X\right)_{t}:=\int_{0}^{t} S_{t-s} \alpha\left(s, X_{s}\right) d s, \quad t \in[0, T], \\
\left(\Phi_{\sigma} X\right)_{t}:=\int_{0}^{t} S_{t-s} \sigma\left(s, X_{s}\right) d W_{s}, \quad t \in[0, T],
\end{gathered}
$$

we have

$$
(\Phi X)_{t}=S_{t} h_{0}+\left(\Phi_{\alpha} X\right)_{t}+\left(\Phi_{\sigma} X\right)_{t}, \quad t \in[0, T]
$$


By the growth estimate (9), we have

$$
\mathbb{E}\left[\int_{0}^{T}\left\|S_{t} h_{0}\right\|^{2 p} d t\right] \leq\left(M e^{\omega T}\right)^{2 p} T \mathbb{E}\left[\left\|h_{0}\right\|^{2 p}\right]<\infty
$$

By Hölder's inequality and the growth estimate (9), we have

$$
\begin{aligned}
& \mathbb{E}\left[\int_{0}^{T}\left\|\left(\Phi_{\alpha} X\right)_{t}\right\|^{2 p} d t\right] \\
& =\mathbb{E}\left[\int_{0}^{T}\left\|\int_{0}^{t} S_{t-s} \alpha\left(s, X_{s}\right) d s\right\|^{2 p} d t\right] \\
& \leq t^{2 p-1} \mathbb{E}\left[\int_{0}^{T} \int_{0}^{t}\left\|S_{t-s} \alpha\left(s, X_{s}\right)\right\|^{2 p} d s d t\right] \\
& \leq T^{2 p-1}\left(M e^{\omega T}\right)^{2 p} \mathbb{E}\left[\int_{0}^{T} \int_{0}^{t}\left\|\alpha\left(s, X_{s}\right)\right\|^{2 p} d s d t\right]
\end{aligned}
$$

and hence, by the linear growth condition (127) and Hölder's inequality, we obtain

$$
\begin{aligned}
& \mathbb{E}\left[\int_{0}^{T}\left\|\left(\Phi_{\alpha} X\right)_{t}\right\|^{2 p} d t\right] \\
& \quad \leq T^{2 p-1}\left(M e^{\omega T} K\right)^{2 p} \mathbb{E}\left[\int_{0}^{T} \int_{0}^{t}\left(1+\left\|X_{s}\right\|\right)^{2 p} d s d t\right] \\
& \quad \leq T^{2 p-1}\left(M e^{\omega T} K\right)^{2 p} 2^{2 p-1} \mathbb{E}\left[\int_{0}^{T} \int_{0}^{t}\left(1+\left\|X_{s}\right\|^{2 p}\right) d s d t\right] \\
& \quad \leq(2 T)^{2 p-1}\left(M e^{\omega T} K\right)^{2 p}\left(\frac{T^{2}}{2}+T \mathbb{E}\left[\int_{0}^{T}\left\|X_{s}\right\|^{2 p} d s\right]\right)<\infty .
\end{aligned}
$$

Furthermore, by Lemma 49 and the growth estimate (9), we have

$$
\begin{aligned}
& \mathbb{E}\left[\int_{0}^{T}\left\|\left(\Phi_{\sigma} X\right)_{t}\right\|^{2 p} d t\right] \\
& =\mathbb{E}\left[\int_{0}^{T}\left\|\int_{0}^{t} S_{t-s} \sigma\left(s, X_{s}\right) d W_{s}\right\|^{2 p} d t\right] \\
& =\int_{0}^{T} \mathbb{E}\left[\left\|\int_{0}^{t} S_{t-s} \sigma\left(s, X_{s}\right) d W_{s}\right\|^{2 p}\right] d t \\
& \leq C_{p} \int_{0}^{T} \mathbb{E}\left[\int_{0}^{t}\left\|S_{t-s} \sigma\left(s, X_{s}\right)\right\|_{L_{2}^{0}(H)}^{2} d s\right]^{p} d t \\
& \leq C_{p}\left(M e^{\omega T}\right)^{2 p} \int_{0}^{T} \mathbb{E}\left[\int_{0}^{t}\left\|\sigma\left(s, X_{s}\right)\right\|_{L_{2}^{0}(H)}^{2} d s\right]^{p} d t,
\end{aligned}
$$

and hence, by the linear growth condition (128) and Hölder's inequality, we obtain

$$
\begin{aligned}
\mathbb{E}\left[\int_{0}^{T}\left\|\left(\Phi_{\sigma} X\right)_{t}\right\|^{2 p} d t\right] \\
\leq C_{p}\left(M e^{\omega T}\right)^{2 p} t^{p-1} \mathbb{E}\left[\int_{0}^{T} \int_{0}^{t}\left\|\sigma\left(s, X_{s}\right)\right\|_{L_{2}^{0}(H)}^{2 p} d s d t\right] \\
\leq C_{p}\left(M e^{\omega T} K\right)^{2 p} T^{p-1} \mathbb{E}\left[\int_{0}^{T} \int_{0}^{t}\left(1+\left\|X_{s}\right\|\right)^{2 p} d s d t\right] \\
\leq C_{p}\left(M e^{\omega T} K\right)^{2 p} T^{p-1} 2^{2 p-1} \mathbb{E}\left[\int_{0}^{T} \int_{0}^{t}\left(1+\left\|X_{s}\right\|^{2 p}\right) d s d t\right] \\
\leq C_{p}\left(M e^{\omega T} K\right)^{2 p} 2^{p}(2 T)^{p-1} \\
\quad \times\left(\frac{T^{2}}{2}+T \mathbb{E}\left[\int_{0}^{T}\left\|X_{s}\right\|^{2 p} d s\right]\right)<\infty .
\end{aligned}
$$

The previous three estimates show that $\Phi X \in L^{2 p}(H)$. Consequently, the mapping $\Phi$ maps $L_{T}^{2 p}(H)$ into itself.

Step 1.3. Now, we show that for some index $n \in \mathbb{N}$ the mapping $\Phi^{n}$ is a contraction on $L_{T}^{2 p}(H)$. Let $X, Y \in L_{T}^{2 p}(H)$, and $t \in[0, T]$ be arbitrary. By Hölder's inequality, the growth estimate (9), and the Lipschitz condition (125) we have

$$
\begin{aligned}
\mathbb{E} & {\left[\left\|\left(\Phi_{\alpha} X\right)_{t}-\left(\Phi_{\alpha} Y\right)_{t}\right\|^{2 p}\right] } \\
& =\mathbb{E}\left[\left\|\int_{0}^{t} S_{t-s} \alpha\left(s, X_{s}\right) d s-\int_{0}^{t} S_{t-s} \alpha\left(s, Y_{s}\right) d s\right\|^{2 p}\right] \\
& =\mathbb{E}\left[\left\|\int_{0}^{t} S_{t-s}\left(\alpha\left(s, X_{s}\right)-\alpha\left(s, Y_{s}\right)\right) d s\right\|^{2 p}\right] \\
& \leq t^{2 p-1} \mathbb{E}\left[\int_{0}^{t}\left\|S_{t-s}\left(\alpha\left(s, X_{s}\right)-\alpha\left(s, Y_{s}\right)\right)\right\|^{2 p} d s\right] \\
& \leq T^{2 p-1}\left(M e^{\omega T}\right)^{2 p} \mathbb{E}\left[\int_{0}^{t}\left\|\alpha\left(s, X_{s}\right)-\alpha\left(s, Y_{s}\right)\right\|^{2 p} d s\right] \\
& \leq T^{2 p-1}\left(M e^{\omega T} L\right)^{2 p} \int_{0}^{t} \mathbb{E}\left[\left\|X_{s}-Y_{s}\right\|^{2 p}\right] d s .
\end{aligned}
$$

Furthermore, by Lemma 49, the growth estimate (9), the Lipschitz condition (126), and Hölder's inequality we obtain

$$
\begin{aligned}
\mathbb{E} & {\left[\left\|\left(\Phi_{\sigma} X\right)_{t}-\left(\Phi_{\sigma} Y\right)_{t}\right\|^{2 p}\right] } \\
& =\mathbb{E}\left[\left\|\int_{0}^{t} S_{t-s} \sigma\left(s, X_{s}\right) d W_{s}-\int_{0}^{t} S_{t-s} \sigma\left(s, Y_{s}\right) d W_{s}\right\|^{2 p}\right] \\
& =\mathbb{E}\left[\left\|\int_{0}^{t} S_{t-s}\left(\sigma\left(s, X_{s}\right)-\sigma\left(s, Y_{s}\right)\right) d W_{s}\right\|^{2 p}\right]
\end{aligned}
$$




$$
\begin{aligned}
& \leq C_{p} \mathbb{E}\left[\int_{0}^{t}\left\|S_{t-s}\left(\sigma\left(s, X_{s}\right)-\sigma\left(s, Y_{s}\right)\right)\right\|_{L_{2}^{0}(H)}^{2} d s\right]^{p} \\
& \leq C_{p}\left(M e^{\omega T}\right)^{2 p} \mathbb{E}\left[\int_{0}^{t}\left\|\sigma\left(s, X_{s}\right)-\sigma\left(s, Y_{s}\right)\right\|_{L_{2}^{0}(H)}^{2} d s\right]^{p} \\
& \leq C_{p}\left(M e^{\omega T} L\right)^{2 p} \int_{0}^{t} \mathbb{E}\left[\left\|X_{s}-Y_{s}\right\|^{2 p}\right] d s .
\end{aligned}
$$

Therefore, defining the constant

$$
C:=2^{2 p-1}\left(T^{2 p-1}\left(M e^{\omega T} L\right)^{2 p}+C_{p}\left(M e^{\omega T} L\right)^{2 p}\right),
$$

by Hölder's inequality, we get

$$
\begin{gathered}
\mathbb{E}\left[\left\|(\Phi X)_{t}-(\Phi Y)_{t}\right\|^{2 p}\right] \\
\leq 2^{2 p-1}\left(\mathbb{E}\left[\left\|\left(\Phi_{\alpha} X\right)_{t}-\left(\Phi_{\alpha} Y\right)_{t}\right\|^{2 p}\right]\right. \\
\left.+\mathbb{E}\left[\left\|\left(\Phi_{\sigma} X\right)_{t}-\left(\Phi_{\sigma} Y\right)_{t}\right\|^{2 p}\right]\right) \\
\leq C \int_{0}^{t} \mathbb{E}\left[\left\|X_{s}-Y_{s}\right\|^{2 p}\right] d s .
\end{gathered}
$$

Thus, by induction for every $n \in \mathbb{N}$, we obtain

$$
\begin{aligned}
& \left\|\Phi^{n} X-\Phi^{n} Y\right\|_{L_{T}^{2 p}(H)} \\
& =\left(\int_{0}^{T} \mathbb{E}\left[\left\|\left(\Phi^{n} X\right)_{t_{1}}-\left(\Phi^{n} Y\right)_{t_{1}}\right\|^{2 p}\right] d t_{1}\right)^{1 / 2 p} \\
& \leq\left(C \int _ { 0 } ^ { T } \left(\int _ { 0 } ^ { t _ { 1 } } \mathbb { E } \left[\|\left(\Phi^{n-1} X\right)_{t_{2}}\right.\right.\right. \\
& \left.\left.\left.-\left(\Phi^{n-1} Y\right)_{t_{2}} \|^{2 p}\right] d t_{2}\right) d t_{1}\right)^{1 / 2 p} \\
& \leq \cdots \leq\left(C^{n} \int_{0}^{T} \int_{0}^{t_{1}} \cdots \int_{0}^{t_{n-1}}\left(\int_{0}^{t_{n}} \mathbb{E}\left[\left\|X_{s}-Y_{s}\right\|^{2 p}\right] d s\right)\right. \\
& \left.\times d t_{n} \cdots d t_{2} d t_{1}\right)^{1 / 2 p} \\
& \leq(C^{n} \underbrace{\left(\int_{0}^{T} \int_{0}^{t_{1}} \cdots \int_{0-1}^{t_{n-1}} 1 d t_{n} \cdots d t_{2} d t_{1}\right)}_{=T^{n} / n !} \\
& \left.\times \mathbb{E}\left[\int_{0}^{T}\left\|X_{s}-Y_{s}\right\|^{2 p} d s\right]\right)^{1 / 2 p} \\
& =\underbrace{\left(\frac{(C T)^{n}}{n !}\right)^{1 / 2 p}}_{\rightarrow 0 \text { for } n \rightarrow \infty}\|X-Y\|_{L_{T}^{2 p}(H)} .
\end{aligned}
$$

Consequently, there exists an index $n \in \mathbb{N}$ such that $\Phi^{n}$ is a contraction, and hence, according to the extension of the Banach fixed point theorem (see Corollary 46), the mapping $\Phi$ has a unique fixed point $X \in L_{T}^{2 p}(H)$. This fixed point $X$ is a solution to the variation of constants equation (130). Since $T \geq 0$ was arbitrary, there exists a process $X$ which is a solution of the variation of constants equation:

$$
X_{t}=S_{t} h_{0}+\int_{0}^{t} S_{t-s} \alpha\left(s, X_{s}\right) d s+\int_{0}^{t} S_{t-s} \sigma\left(s, X_{s}\right) d W_{s},
$$$$
t \geq 0 \text {. }
$$

Step 1.4. In order to prove that $X$ is a mild solution to (44), it remains to show that $X$ has a continuous version. By Lemma 12, the process

$$
t \longmapsto S_{t} h_{0}, \quad t \geq 0,
$$

is continuous, and by Proposition 41 , the process

$$
\int_{0}^{t} S_{t-s} \alpha\left(s, X_{s}\right) d s, \quad t \geq 0,
$$

is continuous, too. Moreover, for every $T \geq 0$, we have, by the linear growth condition (128), Hölder's inequality, and since $X \in L_{T}^{2 P}(H)$, the following estimate:

$$
\begin{aligned}
\mathbb{E} & {\left[\int_{0}^{T}\left\|\sigma\left(s, X_{s}\right)\right\|_{L_{2}^{0}(H)}^{2 p} d s\right] } \\
& \leq K^{2 p} \mathbb{E}\left[\int_{0}^{T}\left(1+\left\|X_{s}\right\|\right)^{2 p} d s\right] \\
& \leq K^{2 p} 2^{2 p-1} \mathbb{E}\left[\int_{0}^{T}\left(1+\left\|X_{s}\right\|^{2 p}\right) d s\right] \\
& =K(2 K)^{2 p-1}\left(T+\mathbb{E}\left[\int_{0}^{T}\left\|X_{s}\right\|^{2 p} d s\right]\right)<\infty .
\end{aligned}
$$

Thus, by Proposition 43 the stochastic convolution $\sigma \star W$ given by

$$
(\sigma \star W)_{t}=\int_{0}^{t} S_{t-s} \sigma\left(s, X_{s}\right) d W_{s}, \quad t \geq 0,
$$

has a continuous version, and consequently, the process $X$ has a continuous version, too. This continuous version is a mild solution to (44).

Step 2. Now let $h_{0}: \Omega \rightarrow H$ be an arbitrary $\mathscr{F}_{0}$-measurable random variable. We define the sequence $\left(h_{n}\right)_{n \in \mathbb{N}}$ of $\mathscr{F}_{0^{-}}$ measurable random variables as

$$
h_{0}^{n}:=h_{0} \mathbb{1}_{\left\{\left\|h_{0}\right\| \leq n\right\}}, \quad n \in \mathbb{N} .
$$

Let $n \in \mathbb{N}$ be arbitrary. Then, as $h_{0}^{n}$ is bounded, we have $\mathbb{E}\left[\left\|h_{0}^{n}\right\|^{2 p}\right]<\infty$ for all $p>1$. By Step 1 the SPDE

$$
\begin{gathered}
d X_{t}^{n}=\left(A X_{t}^{n}+\alpha\left(t, X_{t}^{n}\right)\right) d t+\sigma\left(t, X_{t}^{n}\right) d W_{t}, \\
X_{0}^{n}=h_{0}^{n},
\end{gathered}
$$


has a mild solution $X^{n}$. We define the sequence $\left(\Omega_{n}\right)_{n \in \mathbb{N}} \subset \mathscr{F}_{0}$ as

$$
\Omega_{n}:=\left\{\left\|h_{0}\right\| \leq n\right\}, \quad n \in \mathbb{N} .
$$

Then, we have $\Omega_{n} \subset \Omega_{m}$ for $n \leq m, \Omega=\bigcup_{n \in \mathbb{N}} \Omega_{n}$, and

$$
\Omega_{n} \subset\left\{h_{0}^{n}=h_{0}^{m}\right\} \subset\left\{h_{0}^{n}=h_{0}\right\} \quad \forall n \leq m .
$$

Thus, by Theorem 48 we have (up to indistinguishability)

$$
X^{n} \mathbb{1}_{\Omega_{n}}=X^{m} \mathbb{1}_{\Omega_{n}} \quad \forall n \leq m .
$$

Consequently, the process

$$
X:=\lim _{n \rightarrow \infty} X^{n} \mathbb{1}_{\Omega_{n}}
$$

is a well-defined, continuous, and adapted process, and we have

$$
X^{n} \mathbb{1}_{\Omega_{n}}=X^{m} \mathbb{1}_{\Omega_{n}}=X \mathbb{1}_{\Omega_{n}} \quad \forall n \leq m .
$$

Furthermore, we obtain $\mathbb{P}$-almost surely

$$
\begin{aligned}
& X_{t}=\lim _{n \rightarrow \infty} X_{t}^{n} \mathbb{1}_{\Omega_{n}} \\
& =\lim _{n \rightarrow \infty} \mathbb{1}_{\Omega_{n}}\left(S_{t} h_{0}^{n}+\int_{0}^{t} S_{t-s} \alpha\left(s, X_{s}^{n}\right) d s\right. \\
& \left.+\int_{0}^{t} S_{t-s} \sigma\left(s, X_{s}^{n}\right) d W_{s}\right) \\
& =\lim _{n \rightarrow \infty}\left(S_{t}\left(\mathbb{1}_{\Omega_{n}} h_{0}^{n}\right)+\int_{0}^{t} \mathbb{1}_{\Omega_{n}} S_{t-s} \alpha\left(s, X_{s}^{n}\right) d s\right. \\
& \left.+\int_{0}^{t} \mathbb{1}_{\Omega_{n}} S_{t-s} \sigma\left(s, X_{s}^{n}\right) d W_{s}\right) \\
& =\lim _{n \rightarrow \infty}\left(S_{t}\left(\mathbb{1}_{\Omega_{n}} h_{0}\right)+\int_{0}^{t} \mathbb{1}_{\Omega_{n}} S_{t-s} \alpha\left(s, X_{s}\right) d s\right. \\
& \left.+\int_{0}^{t} \mathbb{1}_{\Omega_{n}} S_{t-s} \sigma\left(s, X_{s}\right) d W_{s}\right) \\
& =\lim _{n \rightarrow \infty} \mathbb{1}_{\Omega_{n}}\left(S_{t} h_{0}+\int_{0}^{t} S_{t-s} \alpha\left(s, X_{s}\right) d s\right. \\
& \left.+\int_{0}^{t} S_{t-s} \sigma\left(s, X_{s}\right) d W_{s}\right) \\
& =S_{t} h_{0}+\int_{0}^{t} S_{t-s} \alpha\left(s, X_{s}\right) d s \\
& +\int_{0}^{t} S_{t-s} \sigma\left(s, X_{s}\right) d W_{s}, \quad t \geq 0,
\end{aligned}
$$

proving that $X$ is a mild solution to (44).

Remark 51. For the proof of Theorem 50, we have used Corollary 46, which is a slight extension of the Banach fixed point theorem. Such an idea has been applied, for example, in [15].
Remark 52. A recent method for proving existence and uniqueness of mild solutions to the SPDE (44) is the method of the moving frame presented in [6]; see also [8]. It allows to reduce SPDE problems to the study of SDEs in infinite dimension. In order to apply this method, we need that the semigroup $\left(S_{t}\right)_{t \geq 0}$ is a semigroup of pseudocontractions.

We close this section with a consequence about the existence of weak solutions.

Corollary 53. Suppose that conditions (125)-(128) are fulfilled. Let $h_{0}: \Omega \rightarrow H$ be a $\mathscr{F}_{0}$-measurable random variable such that $\mathbb{E}\left[\left\|h_{0}\right\|^{2 p}\right]<\infty$ for some $p>1$. Then there exists a (up to indistinguishability) unique weak solution $X$ to (44).

Proof. According to Proposition 35, every weak solution $X$ to (44) is also a mild solution to (44). Therefore, the uniqueness of weak solutions to (44) is a consequence of Theorem 48 .

It remains to prove the existence of a weak solution to (44). Let $T \geq 0$ be arbitrary. By Theorem 50 and its proof, there exists a mild solution $X \in L_{T}^{2 p}(H)$ to (44). By the linear growth condition (128) and Hölder's inequality we obtain

$$
\begin{aligned}
& \mathbb{E}\left[\int_{0}^{T}\left\|\sigma\left(s, X_{s}\right)\right\|_{L_{2}^{0}(H)}^{2}\right] \\
& \quad \leq K^{2} \mathbb{E}\left[\int_{0}^{T}\left(1+\left\|X_{s}\right\|\right)^{2} d s\right] \\
& \quad \leq 2 K^{2} \mathbb{E}\left[\int_{0}^{T}\left(1+\left\|X_{s}\right\|^{2}\right) d s\right] \\
& \quad=2 K^{2}\left(T+\mathbb{E}\left[\int_{0}^{T}\left\|X_{s}\right\|^{2} d s\right]\right) \\
& \quad \leq 2 K^{2}\left(T+T^{1-1 / p} \mathbb{E}\left[\int_{0}^{T}\left\|X_{s}\right\|^{2 p} d s\right]^{1 / p}\right)<\infty
\end{aligned}
$$

showing that condition (72) is fulfilled. Thus, by Proposition 37, the process $X$ is also a weak solution to (44).

\section{Invariant Manifolds for Weak Solutions to SPDEs}

In this section, we deal with invariant manifolds for timehomogeneous SPDEs of the type (44). This topic arises from the natural desire to express the solutions of the SPDE (44), which generally live in the infinite dimensional Hilbert space $H$, by means of a finite dimensional state process and thus to ensure larger analytical tractability. Our goal is to find conditions on the generator $A$ and the coefficients $\alpha, \sigma$ such that for every starting point of a finite dimensional submanifold the solution process stays on the submanifold.

We start with the required preliminaries about finite dimensional submanifolds in Hilbert spaces. In the sequel, let $H$ be a separable Hilbert space.

Definition 54. Let $m, k \in \mathbb{N}$ be positive integers. A subset $\mathscr{M} \subset H$ is called an $m$-dimensional $C^{k}$-submanifold of $H$, 
if for every $h \in \mathscr{M}$ there exist an open neighborhood $U \subset H$ of $h$, an open set $V \subset \mathbb{R}^{m}$, and a mapping $\phi \in C^{2}(V ; H)$ such that

(1) the mapping $\phi: V \rightarrow U \cap \mathscr{M}$ is a homeomorphism;

(2) for all $y \in V$ the mapping $D \phi(y)$ is injective.

The mapping $\phi$ is called a parametrization of $\mathscr{M}$ around $h$.

In what follows, let $\mathscr{M}$ be an $m$-dimensional $C^{k}$ submanifold of $H$.

Lemma 55. Let $\phi_{i}: V_{i} \rightarrow U_{i} \cap \mathscr{M}, i=1,2$ be two parametrizations with $W:=U_{1} \cap U_{2} \cap \mathscr{M} \neq \emptyset$. Then the mapping

$$
\phi_{1}^{-1} \circ \phi_{2}: \phi_{2}^{-1}(W) \longrightarrow \phi_{1}^{-1}(W)
$$

is a $C^{k}$-diffeomorphism.

Proof. See [16, Lemma 6.1.1].

Corollary 56. Let $h \in \mathscr{M}$ be arbitrary, and let $\phi_{i}: V_{i} \rightarrow$ $U_{i} \cap \mathscr{M}, i=1,2$ be two parametrizations of $\mathscr{M}$ around $h$. Then one has

$$
D \phi_{1}\left(y_{1}\right)\left(\mathbb{R}^{m}\right)=D \phi_{2}\left(y_{2}\right)\left(\mathbb{R}^{m}\right),
$$

where $y_{i}=\phi_{i}^{-1}(h)$ for $i=1,2$.

Proof. Since $U_{1}$ and $U_{2}$ are open neighborhoods of $h$, we have $W:=U_{1} \cap U_{2} \cap \mathscr{M} \neq \emptyset$. Thus, by Lemma 55 the mapping

$$
\phi_{1}^{-1} \circ \phi_{2}: \phi_{2}^{-1}(W) \longrightarrow \phi_{1}^{-1}(W)
$$

is a $C^{k}$-diffeomorphism. Using the chain rule, we obtain

$$
\begin{aligned}
D \phi_{2} & \left(y_{2}\right)\left(\mathbb{R}^{m}\right) \\
& =D\left(\phi_{1} \circ\left(\phi_{1}^{-1} \circ \phi_{2}\right)\right)\left(y_{2}\right)\left(\mathbb{R}^{m}\right) \\
& =D \phi_{1}\left(y_{1}\right) D\left(\phi_{1}^{-1} \circ \phi_{2}\right)\left(y_{2}\right)\left(\mathbb{R}^{m}\right) \\
& \subset D \phi_{1}\left(y_{1}\right)\left(\mathbb{R}^{m}\right),
\end{aligned}
$$

and, analogously, we prove that $D \phi_{1}\left(y_{1}\right)\left(\mathbb{R}^{m}\right) \quad \subset$ $D \phi_{2}\left(y_{2}\right)\left(\mathbb{R}^{m}\right)$.

Definition 57. Let $h \in \mathscr{M}$ be arbitrary. The tangent space of $\mathscr{M}$ to $h$ is the subspace

$$
T_{h} \mathscr{M}:=D \phi(y)\left(\mathbb{R}^{m}\right),
$$

where $y=\phi^{-1}(h)$ and $\phi: V \rightarrow U \cap \mathscr{M}$ denotes a parametrization of $\mathscr{M}$ around $h$.

Remark 58. Note that, according to Corollary 56, the Definition 57 of the tangent space $T_{h} \mathscr{M}$ does not depend on the choice of the parametrization $\phi: V \rightarrow U \cap \mathscr{M}$.

Proposition 59. Let $h \in \mathscr{M}$ be arbitrary, and let $\phi: V \rightarrow$ $U \cap \mathscr{M}$ be a parametrization of $\mathscr{M}$ around $h$. Then there exist an open set $V_{0} \subset V$, an open neighborhood $U_{0} \subset U$ of $h$, and a mapping $\widehat{\phi} \in C_{b}^{k}\left(\mathbb{R}^{m} ; H\right)$ with $\left.\phi\right|_{V_{0}}=\left.\widehat{\phi}\right|_{V_{0}}$ such that $\left.\phi\right|_{V_{0}}$ : $V_{0} \rightarrow U_{0} \cap \mathscr{M}$ is a parametrization of $\mathscr{M}$ around $h$, too.
Proof. See [16, Remark 6.1.1].

Remark 60. By Proposition 59 we may assume that any parametrization $\phi: V \rightarrow U \cap \mathscr{M}$ has an extension $\phi \in$ $C_{b}^{k}\left(\mathbb{R}^{m} ; H\right)$.

Proposition 61. Let $D \subset H$ be a dense subset. For every $h_{0} \in$ $\mathscr{M}$ there exist $\zeta_{1}, \ldots, \zeta_{m} \in D$ and a parametrization $\phi: V \rightarrow$ $U \cap \mathscr{M}$ around $h_{0}$ such that

$$
\phi(\langle\zeta, h\rangle)=h \quad \forall h \in U \cap \mathscr{M},
$$

where one uses the notation $\langle\zeta, h\rangle:=\left(\left\langle\zeta_{1}, h\right\rangle, \ldots,\left\langle\zeta_{m}, h\right\rangle\right) \in$ $\mathbb{R}^{m}$.

Proof. See [16, Proposition 6.1.2].

Proposition 62. Let $\phi: V \rightarrow U \cap \mathscr{M}$ be a parametrization as in Proposition 61. Then the following statements are true.

(1) The elements $\zeta_{1}, \ldots, \zeta_{m}$ are linearly independent in $H$.

(2) For every $h \in U \cap \mathscr{M}$, one has the direct sum decomposition

$$
H=T_{h} \mathscr{M} \oplus\left\langle\zeta_{1}, \ldots, \zeta_{m}\right\rangle^{\perp}
$$

(3) For every $h \in U \cap \mathscr{M}$ the mapping

$$
\Pi_{h}=D \phi(y)(\langle\zeta, \bullet\rangle): H \longrightarrow T_{h} \mathscr{M}, \quad \text { where } y=\langle\zeta, h\rangle,
$$

is the corresponding projection according to (166) from $H$ onto $T_{h} \mathscr{M}$, that is, we have

$$
\begin{gathered}
\Pi_{h} \in L(H), \quad \Pi_{h}^{2}=\Pi_{h}, \quad \operatorname{ran}\left(\Pi_{h}\right)=T_{h} \mathscr{M}, \\
\operatorname{ker}\left(\Pi_{h}\right)=\left\langle\zeta_{1}, \ldots, \zeta_{m}\right\rangle^{\perp} .
\end{gathered}
$$

Proof. See [16, Lemma 6.1.3].

From now on, we assume that $\mathscr{M}$ is an $m$-dimensional $C^{2}$-submanifold of $H$.

Proposition 63. Let $\phi: V \rightarrow U \cap \mathscr{M}$ be a parametrization as in Proposition 61. Furthermore, let $\sigma \in C^{1}(H)$ be a mapping such that

$$
\sigma(h) \in T_{h} \mathscr{M} \quad \forall h \in U \cap \mathscr{M}
$$

Then, for every $h \in U \cap \mathscr{M}$ the direct sum decomposition of $D \sigma(h) \sigma(h)$ according to (166) is given by

$$
\begin{aligned}
D \sigma(h) \sigma(h) & \\
= & D \phi(y)(\langle\zeta, D \sigma(h) \sigma(h)\rangle) \\
& +D^{2} \phi(y)(\langle\zeta, \sigma(h)\rangle,\langle\zeta, \sigma(h)\rangle),
\end{aligned}
$$

where $y=\phi^{-1}(h)$. 
Proof. Since $V$ is an open subset of $\mathbb{R}^{m}$, there exists $\epsilon>0$ such that

$$
y+t D \phi(y)^{-1} \sigma(h) \in V \quad \forall t \in(-\epsilon, \epsilon)
$$

Therefore, the curve

$$
c:(-\epsilon, \epsilon) \longrightarrow U \cap \mathscr{M}, \quad c(t):=\phi\left(y+t D \phi(y)^{-1} \sigma(h)\right),
$$

is well defined, and we have $c \in C^{1}((-\epsilon, \epsilon) ; H)$ with $c(0)=h$ and $c^{\prime}(0)=\sigma(h)$. Hence, we have

$$
\left.\frac{d}{d t} \sigma(c(t))\right|_{t=0}=D \sigma(h) \sigma(h) .
$$

Moreover, by condition (169) and Proposition 62, we have

$$
\begin{aligned}
\left.\frac{d}{d t} \sigma(c(t))\right|_{t=0}= & \left.\frac{d}{d t} \Pi_{c(t)} \sigma(c(t))\right|_{t=0} \\
= & \left.\frac{d}{d t} D \phi(\langle\zeta, c(t)\rangle)(\langle\zeta, \sigma(c(t))\rangle)\right|_{t=0} \\
= & D \phi(y)(\langle\zeta, D \sigma(h) \sigma(h)\rangle) \\
& +D^{2} \phi(y)(\langle\zeta, \sigma(h)\rangle,\langle\zeta, \sigma(h)\rangle)
\end{aligned}
$$

The latter two identities prove the desired decomposition (170).

After these preliminaries, we will study invariant manifolds for time-homogeneous SPDEs of the form

$$
\begin{gathered}
d X_{t}=\left(A X_{t}+\alpha\left(X_{t}\right)\right) d t+\sigma\left(X_{t}\right) d W_{t} \\
X_{0}=h_{0},
\end{gathered}
$$

with measurable mappings $\alpha: H \rightarrow H$ and $\sigma: H \rightarrow$ $L_{2}^{0}(H)$. As in the previous sections, the operator $A$ is the infinitesimal generator of a $C_{0}$-semigroup $\left(S_{t}\right)_{t \geq 0}$ on $H$. Note that, by (41), the SPDE (175) can be rewritten equivalently as

$$
\begin{gathered}
d X_{t}=\left(A X_{t}+\alpha\left(X_{t}\right)\right) d t+\sum_{j \in \mathbb{N}} \sigma^{j}\left(X_{t}\right) d \beta_{t}^{j}, \\
X_{0}=h_{0},
\end{gathered}
$$

where $\left(\beta^{j}\right)_{j \in \mathbb{N}}$ denotes the sequence of real-valued independent standard Wiener processes defined in (33) and the mappings $\sigma^{j}: H \rightarrow H, j \in \mathbb{N}$ are given by $\sigma^{j}=\sqrt{\lambda_{j}} \sigma e_{j}$.

For the rest of this section, we assume that there exist a constant $L \geq 0$ such that

$$
\left\|\alpha\left(h_{1}\right)-\alpha\left(h_{2}\right)\right\| \leq L\left\|h_{1}-h_{2}\right\|, \quad h_{1}, h_{2} \in H,
$$

and a sequence $\left(\kappa_{j}\right)_{j \in \mathbb{N}} \subset \mathbb{R}_{+}$with $\sum_{j \in \mathbb{N}} \kappa_{j}^{2}<\infty$ such that for every $j \in \mathbb{N}$ we have

$$
\begin{array}{cl}
\left\|\sigma^{j}\left(h_{1}\right)-\sigma^{j}\left(h_{2}\right)\right\| \leq \kappa_{j}\left\|h_{1}-h_{2}\right\|, & h_{1}, h_{2} \in H, \\
\left\|\sigma^{j}(h)\right\| \leq \kappa_{j}(1+\|h\|), \quad h \in H .
\end{array}
$$

Proposition 64. For every $h_{0} \in H$ there exists a (up to indistinguishability) unique weak solution to (176).

Proof. By (178), for all $h_{1}, h_{2} \in H$ we have

$$
\begin{aligned}
\| \sigma & \left(h_{1}\right)-\sigma\left(h_{2}\right) \|_{L_{2}^{0}(H)} \\
& =\left(\sum_{j \in \mathbb{N}}\left\|\sigma^{j}\left(h_{1}\right)-\sigma^{j}\left(h_{2}\right)\right\|^{2}\right)^{1 / 2} \\
& \leq\left(\sum_{j \in \mathbb{N}} \kappa_{j}^{2}\right)^{1 / 2}\left\|h_{1}-h_{2}\right\| .
\end{aligned}
$$

Moreover, by (177), for every $h \in H$ we have

$$
\begin{aligned}
\|\alpha(h)\| & \leq\|\alpha(h)-\alpha(0)\|+\|\alpha(0)\| \\
& \leq L\|h\|+\|\alpha(0)\| \\
& \leq \max \{L,\|\alpha(0)\|\}(1+\|h\|),
\end{aligned}
$$

and, by (179) we obtain

$$
\begin{aligned}
\|\sigma(h)\|_{L_{2}^{0}(H)} & =\left(\sum_{j \in \mathbb{N}}\left\|\sigma^{j}(h)\right\|^{2}\right)^{1 / 2} \\
& \leq\left(\sum_{j \in \mathbb{N}} \kappa_{j}^{2}\right)^{1 / 2}(1+\|h\|) .
\end{aligned}
$$

Therefore, conditions (125)-(128) are fulfilled, and hence, applying Corollary 53 completes the proof.

Recall that $\mathscr{M}$ denotes a finite dimensional $C^{2}$ submanifold of $H$.

Definition 65. The submanifold $\mathscr{M}$ is called locally invariant for (176), if for every $h_{0} \in \mathscr{M}$ there exists a local weak solution $X$ to (176) with some lifetime $\tau>0$ such that

$$
X_{t \wedge \tau} \in \mathscr{M} \quad \forall t \geq 0, \mathbb{P} \text {-almost surely. }
$$

In order to investigate local invariance of $\mathscr{M}$, we will assume, from now on, that $\sigma^{j} \in C^{1}(H)$ for all $j \in \mathbb{N}$.

Lemma 66. The following statements are true.

(1) For every $h \in H$ one has

$$
\sum_{j \in \mathbb{N}}\left\|D \sigma^{j}(h) \sigma^{j}(h)\right\|<\infty .
$$

(2) The mapping

$$
H \longrightarrow H, \quad h \longmapsto \sum_{j \in \mathbb{N}} D \sigma^{j}(h) \sigma^{j}(h),
$$

is continuous. 
Proof. By (178) and (179), for every $h \in H$ we have

$$
\begin{aligned}
\sum_{j \in \mathbb{N}}\left\|D \sigma^{j}(h) \sigma^{j}(h)\right\| \\
\leq \sum_{j \in \mathbb{N}}\left\|D \sigma^{j}(h)\right\|\left\|\sigma^{j}(h)\right\| \\
\leq(1+\|h\|) \sum_{j \in \mathbb{N}} \kappa_{j}^{2}<\infty,
\end{aligned}
$$

showing (184). Moreover, for every $j \in \mathbb{N}$ the mapping

$$
H \longmapsto H, \quad D \sigma^{j}(h) \sigma^{j}(h),
$$

is continuous, because for all $h_{1}, h_{2} \in H$ we have

$$
\begin{aligned}
& \left\|D \sigma^{j}\left(h_{1}\right) \sigma^{j}\left(h_{1}\right)-D \sigma^{j}\left(h_{2}\right) \sigma^{j}\left(h_{2}\right)\right\| \\
& \leq\left\|D \sigma^{j}\left(h_{1}\right) \sigma^{j}\left(h_{1}\right)-D \sigma^{j}\left(h_{1}\right) \sigma^{j}\left(h_{2}\right)\right\| \\
& \quad+\left\|D \sigma^{j}\left(h_{1}\right) \sigma^{j}\left(h_{2}\right)-D \sigma^{j}\left(h_{2}\right) \sigma^{j}\left(h_{2}\right)\right\| \\
& \leq\left\|D \sigma^{j}\left(h_{1}\right)\right\|\left\|\sigma^{j}\left(h_{1}\right)-\sigma^{j}\left(h_{2}\right)\right\| \\
& \quad+\left\|D \sigma^{j}\left(h_{1}\right)-D \sigma^{j}\left(h_{2}\right)\right\|\left\|\sigma^{j}\left(h_{2}\right)\right\| .
\end{aligned}
$$

Let $v$ be the counting measure on $(\mathbb{N}, \mathfrak{P}(\mathbb{N}))$, which is given by $v(\{j\})=1$ for all $j \in \mathbb{N}$. Then we have

$$
\sum_{j \in \mathbb{N}} D \sigma^{j}(h) \sigma^{j}(h)=\int_{\mathbb{N}} D \sigma^{j}(h) \sigma^{j}(h) \nu(d j) .
$$

Hence, because of the estimate

$$
\left\|D \sigma^{j}(h) \sigma^{j}(h)\right\| \leq(1+\|h\|) \kappa_{j}^{2}, \quad h \in H, j \in \mathbb{N},
$$

the continuity of the mapping (185) is a consequence of Lebesgue's dominated convergence theorem.

For a mapping $\phi \in C_{b}^{2}\left(\mathbb{R}^{m} ; H\right)$ and elements $\zeta_{1}, \ldots, \zeta_{m} \in$ $\mathscr{D}\left(A^{*}\right)$ we define the mappings $\alpha_{\phi, \zeta}: \mathbb{R}^{m} \rightarrow \mathbb{R}^{m}$ and $\sigma_{\phi, \zeta}^{j}:$ $\mathbb{R}^{m} \rightarrow \mathbb{R}^{m}, j \in \mathbb{N}$ as

$$
\begin{gathered}
\alpha_{\phi, \zeta}(y):=\left\langle A^{*} \zeta, \phi(y)\right\rangle+\langle\zeta, \alpha(\phi(y))\rangle, \\
\sigma_{\phi, \zeta}^{j}(y):=\left\langle\zeta, \sigma^{j}(\phi(y))\right\rangle .
\end{gathered}
$$

Proposition 67. Let $\phi \in C_{b}^{2}\left(\mathbb{R}^{m} ; H\right)$ and $\zeta_{1}, \ldots, \zeta_{m} \in \mathscr{D}\left(A^{*}\right)$ be arbitrary. Then, for every $y_{0} \in \mathbb{R}^{m}$ there exists a (up to indistinguishability) unique strong solution to the SDE:

$$
\begin{gathered}
d Y_{t}=\alpha_{\phi, \zeta}\left(Y_{t}\right) d t+\sum_{j \in \mathbb{N}} \sigma_{\phi, \zeta}^{j}\left(Y_{t}\right) d \beta_{t}^{j}, \\
Y_{0}=y_{0} .
\end{gathered}
$$

Proof. By virtue of the assumption $\phi \in C_{b}^{2}\left(\mathbb{R}^{m} ; H\right)$ and (177)(179), there exist a constant $\widetilde{L} \geq 0$ such that

$$
\left\|\alpha_{\phi, \zeta}\left(y_{1}\right)-\alpha_{\phi, \zeta}\left(y_{2}\right)\right\|_{\mathbb{R}^{m}} \leq \widetilde{L}\left\|y_{1}-y_{2}\right\|_{\mathbb{R}^{m}}, \quad y_{1}, y_{2} \in \mathbb{R}^{m},
$$

and a sequence $\left(\widetilde{\kappa}_{j}\right)_{j \in \mathbb{N}} \subset \mathbb{R}_{+}$with $\sum_{j \in \mathbb{N}} \widetilde{\kappa}_{j}^{2}<\infty$ such that for every $j \in \mathbb{N}$ we have

$$
\begin{gathered}
\left\|\sigma_{\phi, \zeta}^{j}\left(y_{1}\right)-\sigma_{\phi, \zeta}^{j}\left(y_{2}\right)\right\|_{\mathbb{R}^{m}} \leq \widetilde{\kappa}_{j}\left\|y_{1}-y_{2}\right\|_{\mathbb{R}^{m}}, \quad y_{1}, y_{2} \in \mathbb{R}^{m}, \\
\left\|\sigma_{\phi, \zeta}^{j}(y)\right\|_{\mathbb{R}^{m}} \leq \widetilde{\kappa}_{j}\left(1+\|y\|_{\mathbb{R}^{m}}\right), \quad y \in \mathbb{R}^{m} .
\end{gathered}
$$

Therefore, by Proposition 64, for every $y_{0} \in \mathbb{R}^{m}$ there exists a (up to indistinguishability) unique weak solution to (192), which, according to Proposition 39 is also a strong solution to (192). The uniqueness of strong solutions to (192) is a consequence of Proposition 39 and Theorem 48.

Now, we are ready to formulate and prove our main result of this section.

Theorem 68. The following statements are equivalent.

(1) The submanifold $\mathscr{M}$ is locally invariant for (176).

(2) One has

$$
\begin{gathered}
\mathscr{M} \subset \mathscr{D}(A), \\
\sigma^{j}(h) \in T_{h} \mathscr{M} \quad \forall h \in \mathscr{M}, \text { all } j \in \mathbb{N}, \\
A h+\alpha(h)-\frac{1}{2} \sum_{j \in \mathbb{N}} D \sigma^{j}(h) \sigma^{j}(h) \in T_{h} \mathscr{M} \quad \forall h \in \mathscr{M} .
\end{gathered}
$$

(3) The operator $A$ is continuous on $\mathscr{M}$, and for each $h_{0} \in$ $\mathscr{M}$ there exists a local strong solution $X$ to (176) with some lifetime $\tau>0$ such that

$$
X_{t \wedge \tau} \in \mathscr{M} \quad \forall t \geq 0, \mathbb{P} \text {-almost surely. }
$$

Proof. (1) $\Rightarrow(2)$ : Let $h \in \mathscr{M}$ be arbitrary. By Proposition 61 and Remark 60 there exist elements $\zeta_{1}, \ldots, \zeta_{m} \in \mathscr{D}\left(A^{*}\right)$ and a parametrization $\phi: V \rightarrow U \cap \mathscr{M}$ around $h$ such that the inverse $\phi^{-1}: U \cap \mathscr{M} \rightarrow V$ is given by $\phi^{-1}=\langle\zeta, \bullet\rangle$, and $\phi$ has an extension $\phi \in C_{b}^{2}\left(\mathbb{R}^{m} ; H\right)$. Since the submanifold $\mathscr{M}$ is locally invariant for (176), there exists a local weak solution $X$ to (176) with initial condition $h$ and some lifetime $\varrho>0$ such that

$$
X_{t \wedge \varrho} \in \mathscr{M} \quad \forall t \geq 0, \mathbb{P} \text {-almost surely. }
$$

Since $U$ is an open neighborhood of $h$, there exists $\epsilon>0$ such that $\overline{B_{\epsilon}(h)} \subset U$, where $B_{\epsilon}(h)$ denotes the open ball:

$$
B_{\epsilon}(h)=\{g \in H:\|g-h\|<\epsilon\} .
$$

We define the stopping time

$$
\tau:=\varrho \wedge \inf \left\{t \geq 0: X_{t} \notin B_{\epsilon}(h)\right\} .
$$

Since the process $X$ has continuous sample paths and satisfies $X_{0}=h$, we have $\tau>0$ and $\mathbb{P}$-almost surely

$$
X_{t \wedge \tau} \in U \cap \mathscr{M} \quad \forall t \geq 0 .
$$


Defining the $\mathbb{R}^{m}$-valued process $Y:=\langle\zeta, X\rangle$ we have $\mathbb{P}$ almost surely

$$
Y_{t \wedge \tau} \in V \quad \forall t \geq 0
$$

Moreover, since $X$ is a weak solution to (176) with initial condition $h$, setting $y:=\langle\zeta, h\rangle \in V$ we have $\mathbb{P}$-almost surely

$$
\begin{aligned}
Y_{t \wedge \tau}= & \langle\zeta, h\rangle+\int_{0}^{t \wedge \tau}\left(\left\langle A^{*} \zeta, X_{s}\right\rangle+\left\langle\zeta, \alpha\left(X_{s}\right)\right\rangle\right) d s \\
& +\sum_{j \in \mathbb{N}} \int_{0}^{t \wedge \tau}\left\langle\zeta, \sigma^{j}\left(X_{s}\right)\right\rangle d \beta_{s}^{j} \\
= & \langle\zeta, h\rangle+\int_{0}^{t \wedge \tau} \alpha_{\phi, \zeta}\left(\left\langle\zeta, X_{s}\right\rangle\right) d s \\
& +\sum_{j \in \mathbb{N}} \int_{0}^{t \wedge \tau} \sigma_{\phi, \zeta}^{j}\left(\left\langle\zeta, X_{s}\right\rangle\right) d \beta_{s}^{j} \\
= & y+\int_{0}^{t \wedge \tau} \alpha_{\phi, \zeta}\left(Y_{s}\right) d s \\
& +\sum_{j \in \mathbb{N}} \int_{0}^{t \wedge \tau} \sigma_{\phi, \zeta}^{j}\left(Y_{s}\right) d \beta_{s}^{j}, \quad t \geq 0,
\end{aligned}
$$

showing that $Y$ is a local strong solution to (192) with initial condition $y$. By Itô's formula (Theorem 26) we obtain $\mathbb{P}$ almost surely

$$
\begin{aligned}
X_{t \wedge \tau}= & \phi\left(Y_{t \wedge \tau}\right) \\
= & h+\int_{0}^{t \wedge \tau}\left(D \phi\left(Y_{s}\right) \alpha_{\phi, \zeta}\left(Y_{s}\right)\right. \\
& \left.+\frac{1}{2} \sum_{j \in \mathbb{N}} D^{2} \phi\left(Y_{s}\right)\left(\sigma_{\phi, \zeta}^{j}\left(Y_{s}\right), \sigma_{\phi, \zeta}^{j}\left(Y_{s}\right)\right)\right) d s \\
& +\sum_{j \in \mathbb{N}} \int_{0}^{t \wedge \tau} D \phi\left(Y_{s}\right) \sigma_{\phi, \zeta}^{j}\left(Y_{s}\right) d \beta_{s}^{j}, \quad t \geq 0 .
\end{aligned}
$$

Now, let $\xi \in \mathscr{D}\left(A^{*}\right)$ be arbitrary. Then we have $\mathbb{P}$-almost surely

$$
\begin{aligned}
& \left\langle\xi, X_{t \wedge \tau}\right\rangle \\
& =\langle\xi, h\rangle \\
& \quad+\int_{0}^{t \wedge \tau}\left\langle\xi, D \phi\left(Y_{s}\right) \alpha_{\phi, \zeta}\left(Y_{s}\right)\right. \\
& \left.\quad+\frac{1}{2} \sum_{j \in \mathbb{N}} D^{2} \phi\left(Y_{s}\right)\left(\sigma_{\phi, \zeta}^{j}\left(Y_{s}\right), \sigma_{\phi, \zeta}^{j}\left(Y_{s}\right)\right)\right\rangle d s \\
& \quad+\sum_{j \in \mathbb{N}} \int_{0}^{t \wedge \tau}\left\langle\xi, D \phi\left(Y_{s}\right) \sigma_{\phi, \zeta}^{j}\left(Y_{s}\right)\right\rangle d \beta_{s}^{j}, \quad t \geq 0 .
\end{aligned}
$$

On the other hand, since $X$ is a local weak solution to (176) with initial condition $h$ and lifetime $\tau$, we have $\mathbb{P}$-almost surely for all $t \geq 0$ the identity

$$
\begin{aligned}
\left\langle\xi, X_{t \wedge \tau}\right\rangle= & \langle\xi, h\rangle+\int_{0}^{t \wedge \tau}\left(\left\langle A^{*} \xi, X_{s}\right\rangle+\left\langle\xi, \alpha\left(X_{s}\right)\right\rangle\right) d s \\
& +\sum_{j \in \mathbb{N}} \int_{0}^{t \wedge \tau}\left\langle\xi, \sigma^{j}\left(X_{s}\right)\right\rangle d \beta_{s}^{j} .
\end{aligned}
$$

Combining (206) and (207) yields up to indistinguishability

$$
B+M=0
$$

where the processes $B$ and $M$ are defined as

$$
\begin{aligned}
& B_{t}:=\int_{0}^{t \wedge \tau}\left(\left\langle A^{*} \xi, X_{s}\right\rangle+\langle\xi, \alpha\right.\left(X_{s}\right)-D \phi\left(Y_{s}\right) \alpha_{\phi, \zeta}\left(Y_{s}\right) \\
&-\frac{1}{2} \sum_{j \in \mathbb{N}} D^{2} \phi\left(Y_{s}\right) \\
&\left.\left.\times\left(\sigma_{\phi, \zeta}^{j}\left(Y_{s}\right), \sigma_{\phi, \zeta}^{j}\left(Y_{s}\right)\right)\right\rangle\right) d s \\
& M_{t}:=\sum_{j \in \mathbb{N}} \int_{0}^{t \wedge \tau}\left\langle\xi, \sigma^{j}\left(X_{s}\right)-\right.\left.D \phi\left(Y_{s}\right) \sigma_{\phi, \zeta}^{j}\left(Y_{s}\right)\right\rangle d \beta_{s}^{j}, \\
& t \geq 0 .
\end{aligned}
$$

The process $B+M$ is a continuous semimartingale with canonical decomposition (208). Since the canonical decomposition of a continuous semimartingale is unique up to indistinguishability, we deduce that $B=M=0$ up to indistinguishability. Using the Itô isometry (39) we obtain $\mathbb{P}$ almost surely

$$
\begin{aligned}
& \int_{0}^{t \wedge \tau}\left(\left\langle A^{*} \xi, X_{s}\right\rangle\right. \\
& +\left\langle\xi, \alpha\left(X_{s}\right)-D \phi\left(Y_{s}\right) \alpha_{\phi, \zeta}\left(Y_{s}\right)\right. \\
& \left.\left.\quad-\frac{1}{2} \sum_{j \in \mathbb{N}} D^{2} \phi\left(Y_{s}\right)\left(\sigma_{\phi, \zeta}^{j}\left(Y_{s}\right), \sigma_{\phi, \zeta}^{j}\left(Y_{s}\right)\right)\right\rangle\right) d s \\
& =0, \quad t \geq 0, \\
& \int_{0}^{t \wedge \tau} \sum_{j \in \mathbb{N}}\left|\left\langle\xi, \sigma^{j}\left(X_{s}\right)-D \phi\left(Y_{s}\right) \sigma_{\phi, \zeta}^{j}\left(Y_{s}\right)\right\rangle\right|^{2} d s=0,
\end{aligned}
$$$$
t \geq 0
$$ 
By the continuity of the processes $X$ and $Y$ we obtain for all $\xi \in \mathscr{D}\left(A^{*}\right)$ the following identities:

$$
\begin{gathered}
\left\langle A^{*} \xi, h\right\rangle+\left\langle\xi, \alpha(h)-D \phi(y) \alpha_{\phi, \zeta}(y)\right. \\
\left.\quad-\frac{1}{2} \sum_{j \in \mathbb{N}} D^{2} \phi(y)\left(\sigma_{\phi, \zeta}^{j}(y), \sigma_{\phi, \zeta}^{j}(y)\right)\right\rangle=0, \\
\left\langle\xi, \sigma^{j}(h)-D \phi(y) \sigma_{\phi, \zeta}^{j}(y)\right\rangle=0, \quad j \in \mathbb{N} .
\end{gathered}
$$

Consequently, the mapping $\xi \mapsto\left\langle A^{*} \xi, h\right\rangle$ is continuous on $\mathscr{D}\left(A^{*}\right)$, and hence we have $h \in \mathscr{D}\left(A^{* *}\right)$ by the definition of the domain provided in (4). By Proposition 7 we have $A=A^{* *}$, and thus we obtain $h \in \mathscr{D}(A)$, proving (195). By Proposition 7, the domain $\mathscr{D}\left(A^{*}\right)$ is dense in $H$, and thus

$$
\sigma^{j}(h)=D \phi(y) \sigma_{\phi, \zeta}^{j}(y) \in T_{h} \mathscr{M}, j \in \mathbb{N},
$$

showing (196). Moreover, for all $\xi \in \mathscr{D}\left(A^{*}\right)$ we have

$$
\begin{aligned}
& \left\langle\xi, A h+\alpha(h)-D \phi(y) \alpha_{\phi, \zeta}(y)\right. \\
& \left.\quad-\frac{1}{2} \sum_{j \in \mathbb{N}} D^{2} \phi(y)\left(\sigma_{\phi, \zeta}^{j}(y), \sigma_{\phi, \zeta}^{j}(y)\right)\right\rangle=0 .
\end{aligned}
$$

Since the domain $\mathscr{D}\left(A^{*}\right)$ is dense in $H$, together with Proposition 63, we obtain

$$
\begin{gathered}
A h+\alpha(h)-\frac{1}{2} \sum_{j \in \mathbb{N}} D \sigma^{j}(h) \sigma^{j}(h) \\
=A h+\alpha(h)-\frac{1}{2} \sum_{j \in \mathbb{N}}\left(D \phi(y)\left(\left\langle\zeta, D \sigma^{j}(h) \sigma^{j}(h)\right\rangle\right)\right. \\
\left.+D^{2} \phi(y)\left(\sigma_{\phi, \zeta}^{j}(y), \sigma_{\phi, \zeta}^{j}(y)\right)\right) \\
=D \phi(y) \alpha_{\phi, \zeta}(y)-\frac{1}{2} \sum_{j \in \mathbb{N}} D \phi(y)\left(\left\langle\zeta, D \sigma^{j}(h) \sigma^{j}(h)\right\rangle\right) \\
=D \phi(y)\left(\alpha_{\phi, \zeta}(y)-\frac{1}{2} \sum_{j \in \mathbb{N}}\left\langle\zeta, D \sigma^{j}(h) \sigma^{j}(h)\right\rangle\right) \in T_{h} \mathscr{M},
\end{gathered}
$$

which proves (197).

$(2) \Rightarrow(1)$ : Let $h_{0} \in \mathscr{M}$ be arbitrary. By Proposition 61 and Remark 60 there exist $\zeta_{1}, \ldots, \zeta_{m} \in \mathscr{D}\left(A^{*}\right)$ and a parametrization $\phi: V \rightarrow U \cap \mathscr{M}$ around $h_{0}$ such that the inverse $\phi^{-1}: U \cap \mathscr{M} \rightarrow V$ is given by $\phi^{-1}=\langle\zeta, \bullet\rangle$, and $\phi$ has an extension $\phi \in C_{b}^{2}\left(\mathbb{R}^{m} ; H\right)$. Let $h \in U \cap \mathscr{M}$ be arbitrary, and set $y:=\langle\zeta, h\rangle \in V$. By relations (195), (197) and Proposition 62, we obtain

$$
\begin{aligned}
A h & +\alpha(h)-\frac{1}{2} \sum_{j \in \mathbb{N}} D \sigma^{j}(h) \sigma^{j}(h) \\
& =D \phi(y)\left(\left\langle\zeta, A h+\alpha(h)-\frac{1}{2} \sum_{j \in \mathbb{N}} D \sigma^{j}(h) \sigma^{j}(h)\right\rangle\right),
\end{aligned}
$$

and thus

$$
\begin{aligned}
A h=D \phi(y)\left(\left\langle A^{*} \zeta, h\right\rangle\right. \\
\\
\left.+\left\langle\zeta, \alpha(h)-\frac{1}{2} \sum_{j \in \mathbb{N}} D \sigma^{j}(h) \sigma^{j}(h)\right\rangle\right) \\
-\alpha(h)+\frac{1}{2} \sum_{j \in \mathbb{N}} D \sigma^{j}(h) \sigma^{j}(h) .
\end{aligned}
$$

Together with Lemma 66, this proves the continuity of $A$ on $U \cap \mathscr{M}$. Since $h_{0} \in \mathscr{M}$ was arbitrary, this proves that $A$ is continuous on $\mathscr{M}$.

Furthermore, by (196) and Proposition 62 we have

$$
\sigma^{j}(h)=D \phi(y) \sigma_{\phi, \zeta}^{j}(h) \quad \text { for every } j \in \mathbb{N} .
$$

Moreover, by (195), (197), and Propositions 62 and 63, we obtain

$$
\begin{aligned}
A h+\alpha(h)-\frac{1}{2} \sum_{j \in \mathbb{N}} D \sigma^{j}(h) \sigma^{j}(h) \\
=D \phi(y)(\langle\zeta, A h+\alpha(h) \\
\left.\left.\left.=-\frac{1}{2} \sum_{j \in \mathbb{N}} D \sigma^{j}(h) \sigma^{j}(h)\right\rangle\right)\right) \\
=\frac{1}{2} \sum_{j \in \mathbb{N}} D \phi(y)\left\langle\zeta, D \sigma^{j}(h) \sigma^{j}(h)\right\rangle \\
=D \phi(y) \alpha_{\phi, \zeta}(y) \\
+\frac{1}{2} \sum_{j \in \mathbb{N}}\left(D^{2} \phi(y)\left(A_{\phi, \zeta}^{j}(y), \sigma_{\phi, \zeta}^{j}(y)\right)\right. \\
\left.-D \sigma^{j}(h) \sigma^{j}(h)\right) .
\end{aligned}
$$

This gives us

$$
\begin{aligned}
A h+\alpha(h)= & D \phi(y) \alpha_{\phi, \zeta}(y) \\
& +\frac{1}{2} \sum_{j \in \mathbb{N}} D^{2} \phi(y)\left(\sigma_{\phi, \zeta}^{j}(y), \sigma_{\phi, \zeta}^{j}(y)\right) .
\end{aligned}
$$


Now, let $Y$ be the strong solution to (192) with initial condition $y_{0}:=\left\langle\zeta, h_{0}\right\rangle \in V$. Since $V$ is open, there exists $\epsilon>0$ such that $\overline{B_{\epsilon}\left(y_{0}\right)} \subset V$. We define the stopping time

$$
\tau:=\inf \left\{t \geq 0: Y_{t} \notin B_{\epsilon}\left(y_{0}\right)\right\} .
$$

Since the process $Y$ has continuous sample paths and satisfies $Y_{0}=y_{0}$, we have $\tau>0$ and $\mathbb{P}$-almost surely

$$
Y_{t \wedge \tau} \in V \quad \forall t \geq 0 .
$$

Therefore, defining the $H$-valued process $X:=\phi(Y)$, we have $\mathbb{P}$-almost surely

$$
X_{t \wedge \tau} \in U \cap \mathscr{M} \quad \forall t \geq 0 .
$$

Moreover, using Itô's formula (Theorem 26) and incorporating (217), (219), we obtain $\mathbb{P}$-almost surely

$$
\begin{aligned}
X_{t \wedge \tau}= & \phi\left(y_{0}\right) \\
& +\int_{0}^{t \wedge \tau}\left(D \phi\left(Y_{s}\right) \alpha_{\phi, \zeta}\left(Y_{s}\right)\right. \\
& +\frac{1}{2} \sum_{j \in \mathbb{N}} D^{2} \phi\left(Y_{s}\right) \phi\left(Y_{s}\right) \\
& \left.\times\left(\sigma_{\phi, \zeta}^{j}\left(Y_{s}\right), \sigma_{\phi, \zeta}^{j}\left(Y_{s}\right)\right)\right) d s \\
& +\sum_{j \in \mathbb{N}} \int_{0}^{t \wedge \tau} D \phi\left(Y_{s}\right) \sigma_{\phi, \zeta}^{j}\left(Y_{s}\right) d \beta_{s}^{j} \\
= & \phi\left(y_{0}\right)+\int_{0}^{t \wedge \tau}\left(A \phi\left(Y_{s}\right)+\alpha\left(\phi\left(Y_{s}\right)\right)\right) d s \\
& +\sum_{j \in \mathbb{N}} \int_{0}^{t \wedge \tau} \sigma^{j}\left(\phi\left(Y_{s}\right)\right) d \beta_{s}^{j} \\
= & h_{0}+\int_{0}^{t \wedge \tau}\left(A X_{s}+\alpha\left(X_{s}\right)\right) d s \\
& +\sum_{j \in \mathbb{N}} \int_{0}^{t \wedge \tau} \sigma^{j}\left(X_{s}\right) d \beta_{s}^{j}, \quad t \geq 0,
\end{aligned}
$$

showing that $X$ is a local strong solution to (44) with lifetime $\tau$.

(3) $\Rightarrow(1)$ : This implication is a direct consequence of Proposition 29.

The results from this section are closely related to the existence of finite dimensional realizations, that is, the existence of invariant manifolds for each starting point $h_{0}$, and we point out the papers [17-22] regarding this topic. Furthermore, we mention that Theorem 68 has been extended in [23] to SPDEs with jumps.

\section{Acknowledgments}

The author is grateful to Daniel Gaigall, Georg Grafendorfer, Florian Modler, and Thomas Salfeld for the valuable comments and discussions.

\section{References}

[1] G. Da Prato and J. Zabczyk, Stochastic Equations in Infinite Dimensions, Cambridge University Press, Cambridge, UK, 1992.

[2] C. Prévôt and M. Röckner, A Concise Course on Stochastic Partial Differential Equations, Springer, Berlin, Germany, 2007.

[3] L. Gawarecki and V. Mandrekar, Stochastic Differential Equations in Infinite Dimensions with Applications to Stochastic Partial Differential Equations, Springer, Berlin, Germany, 2011.

[4] S. Peszat and J. Zabczyk, Stochastic Partial Differential Equations with Lévy Noise, Cambridge University Press, Cambridge, UK, 2007.

[5] S. Albeverio, V. Mandrekar, and B. Rüdiger, "Existence of mild solutions for stochastic differential equations and semilinear equations with non-Gaussian Lévy noise," Stochastic Processes and Their Applications, vol. 119, no. 3, pp. 835-863, 2009.

[6] D. Filipović, S. Tappe, and J. Teichmann, "Jump-diffusions in Hilbert spaces: existence, stability and numerics," Stochastics, vol. 82, no. 5, pp. 475-520, 2010.

[7] C. Marinelli, C. Prévôt, and M. Röckner, "Regular dependence on initial data for stochastic evolution equations with multiplicative Poisson noise," Journal of Functional Analysis, vol. 258, no. 2, pp. 616-649, 2010.

[8] S. Tappe, "Some refinements of existence results for SPDEs driven by Wiener processes and Poisson random measures," International Journal of Stochastic Analysis, vol. 2012, Article ID 236327, 24 pages, 2012.

[9] T. Nakayama, "Support theorem for mild solutions of SDE's in Hilbert spaces," Journal of Mathematical Sciences, the University of Tokyo, vol. 11, no. 3, pp. 245-311, 2004.

[10] T. Nakayama, "Viability theorem for SPDE's including HJM framework," Journal of Mathematical Sciences, the University of Tokyo, vol. 11, no. 3, pp. 313-324, 2004.

[11] W. Rudin, Functional Analysis, McGraw-Hill, New York, NY, USA, 2nd edition, 1991.

[12] D. Werner, Funktionalanalysis, Springer, Berlin, Germany, 2007.

[13] C. D. Aliprantis and K. C. Border, Infinite Dimensional Analysis, Springer, Berlin, Germany, 3rd edition, 2006.

[14] S. N. Ethier and T. G. Kurtz, Markov Processes. Characterization and Convergence, Wiley Series in Probability and Statistics, John Wiley \& Sons, New Jersey, NJ, USA, 1986.

[15] V. Mandrekar and B. Rüdiger, "Existence and uniqueness of path wise solutions for stochastic integral equations driven by Lévy noise on separable Banach spaces," Stochastics, vol. 78, no. 4, pp. 189-212, 2006.

[16] D. Filipović, Consistency Problems for Heath-Jarrow-Morton Interest Rate Models, Springer, Berlin, Germany, 2001.

[17] T. Björk and L. Svensson, "On the existence of finitedimensional realizations for nonlinear forward rate models," Mathematical Finance, vol. 11, no. 2, pp. 205-243, 2001.

[18] T. Björk and C. Landén, "On the construction of finite dimensional realizations for nonlinear forward rate models," Finance and Stochastics, vol. 6, no. 3, pp. 303-331, 2002.

[19] D. Filipović and J. Teichmann, "Existence of invariant manifolds for stochastic equations in infinite dimension," Journal of Functional Analysis, vol. 197, no. 2, pp. 398-432, 2003.

[20] D. Filipović and J. Teichmann, "On the geometry of the term structure of interest rates," Proceedings of The Royal Society of London A, vol. 460, no. 2041, pp. 129-167, 2004.

[21] S. Tappe, "An alternative approach on the existence of affine realizations for HJM term structure models," Proceedings of 
The Royal Society of London A, vol. 466, no. 2122, pp. 3033-3060, 2010.

[22] S. Tappe, "Existence of affine realizations for Lévy term structure models," Proceedings of The Royal Society of London A, vol. 468, no. 2147, pp. 3685-3704, 2012.

[23] D. Filipovic, S. Tappe, and J. Teichmann, "Invariant manifolds with boundary for jump-diffusions," http://arxiv.org/abs/1202 .1076 . 


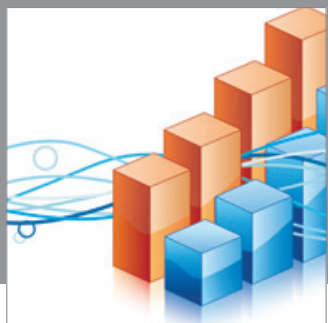

Advances in

Operations Research

mansans

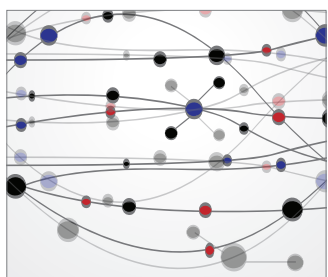

The Scientific World Journal
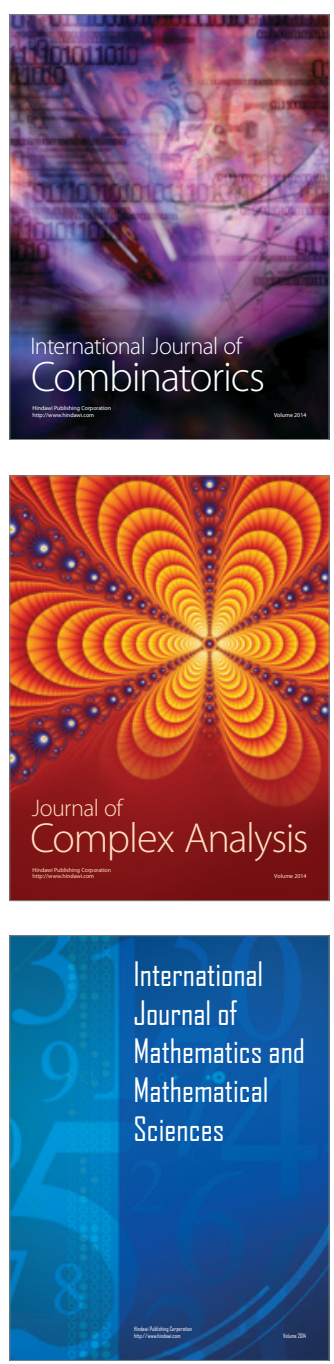
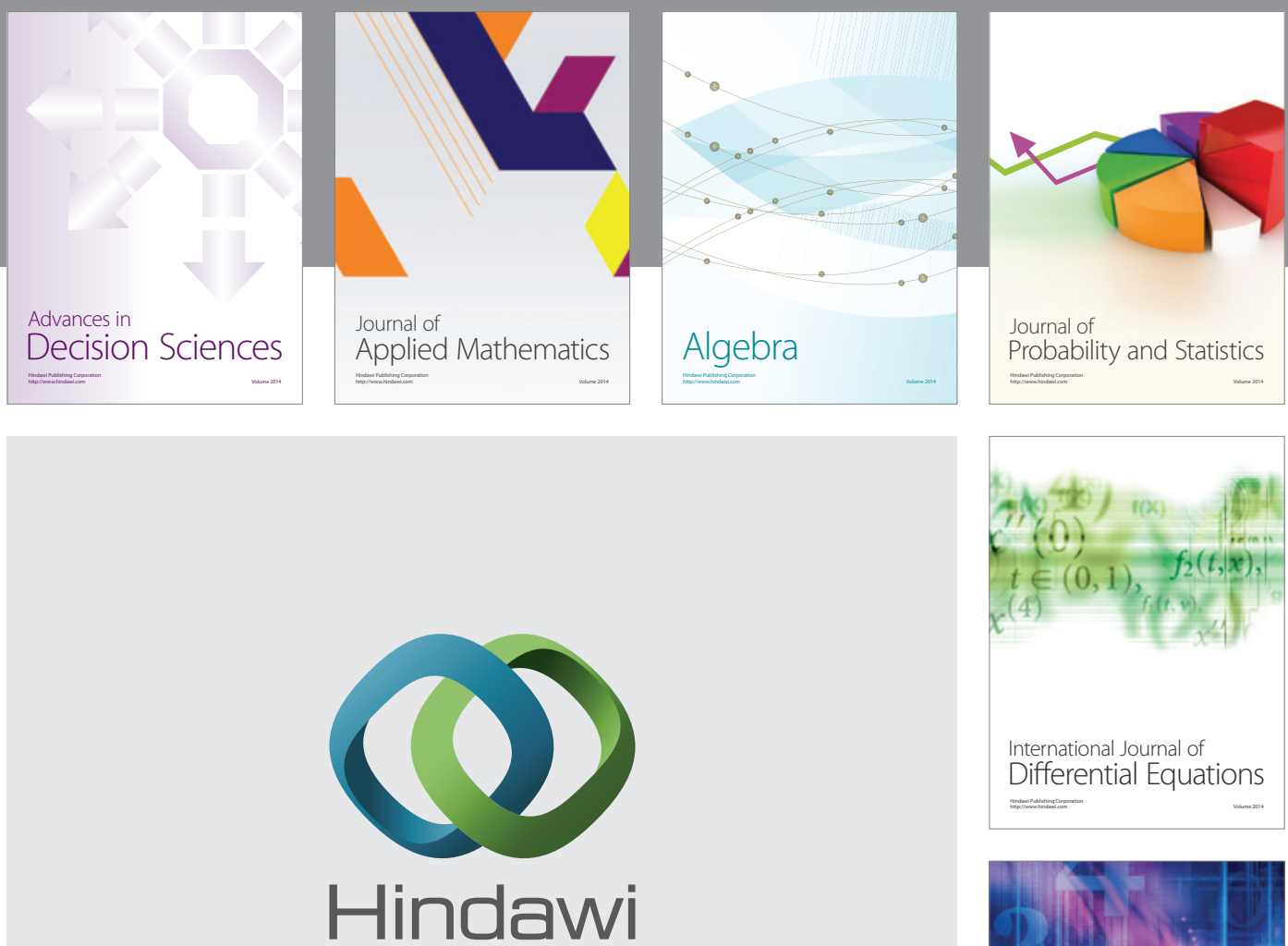

Submit your manuscripts at http://www.hindawi.com
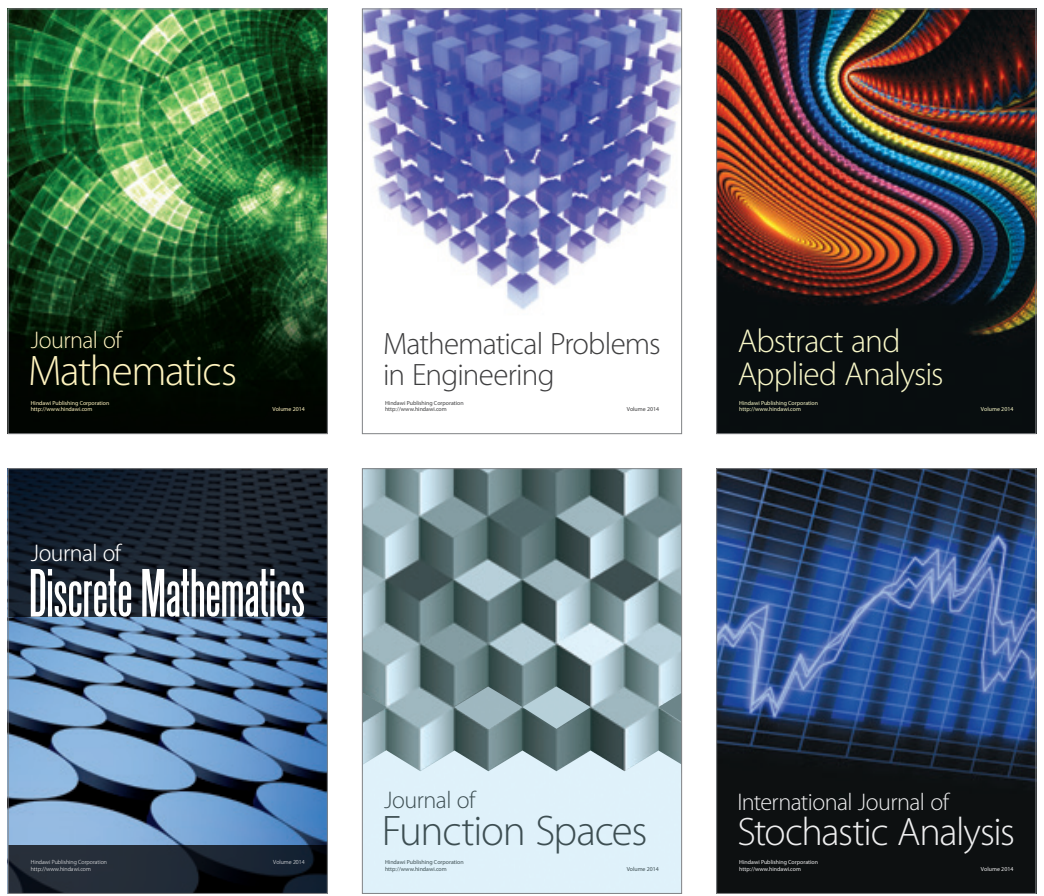

Journal of

Function Spaces

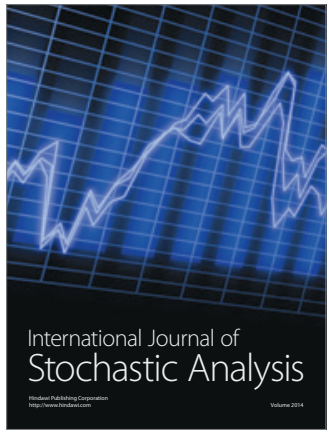

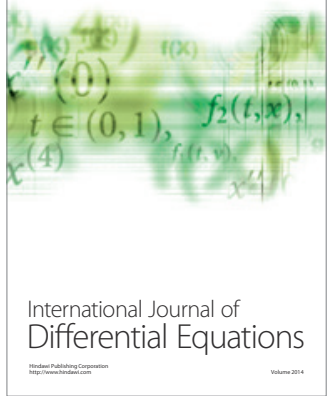
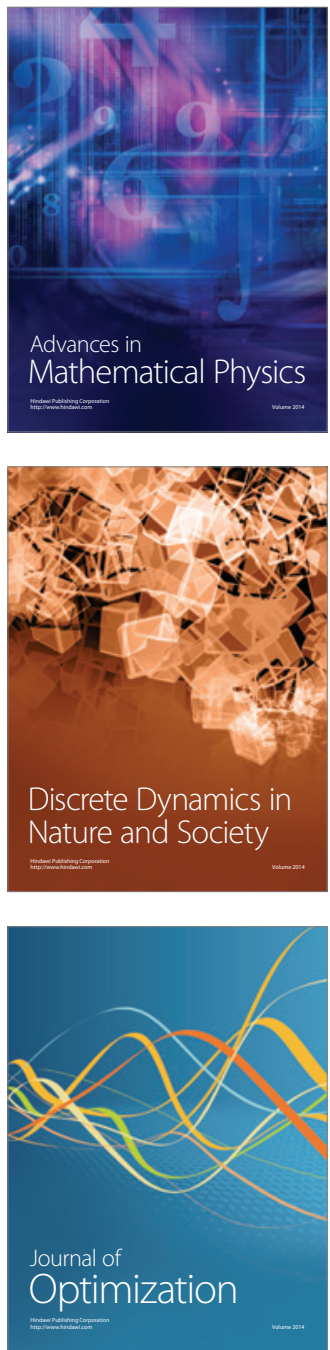